\title{
Eletroencefalograma Processado em Crianças Anestesiadas com Sevoflurano. É Possível? *
}

\section{Processed Electroencephalogram in Children Anesthetized with Sevoflurane. Is it Feasible?}

Rogean Rodrigues Nunes, TSA ${ }^{1}$; Sara Lúcia Cavalcante, TSA ${ }^{2}$; Alberto Esteves Gemal, TSA ${ }^{3}$; Domingos Gerson de Sabóia Amorim, TSA ${ }^{4}$

\begin{abstract}
RESUMO
Nunes RR, Cavalcante SL, Gemal AE, Amorim DGS - Eletroencefalograma Processado em Crianças Anestesiadas com Sevoflurano. É Possível?
\end{abstract}

JUSTIFICATIVA E OBJETIVOS: O índice bispectral (BIS) tem sido indicado como um substrato importante na mensuração do efeito hipnótico de drogas anestésicas. No entanto, existem apenas dados limitados do uso do EEG em crianças durante anestesia. O objetivo deste estudo é avaliar, em crianças, as mudanças no BIS, SEF ${ }_{95 \%}$, amplitude relativa na banda de freqüência delta ( $\delta \%$ ) e taxa de supressão de surtos (TS), correlacionando com variáveis farmacodinâmicas do sevoflurano (CE e CE/CAM), comparando-as com o adulto.

MÉTODO: Participaram do estudo, 100 pacientes de ambos os sexos, com idades entre 0 e 40 anos, estado físico ASA I e II. Todos os pacientes foram induzidos com sevoflurano, sendo utilizado bloqueador neuromuscular quando o BIS atingiu 30, sendo estratificados em 5 grupos: GI (20) - idade entre 0 e 6 meses; GII (20) - idade > 6 meses até 2 anos; GIII (20) - idade > 2 anos até 12 anos; GIV (20) - idade > 12 anos até 18 anos e GV (20) - idade > 18 anos até 40 anos. Em cada grupo, 5 momentos foram avaliados: $M_{1}$ (alerta); $M_{2}$ (BIS 60); $M_{3}$ (BIS 50); $M_{4}$ (BIS 40) e $M_{5}$ (despertar), sendo, em todos os momentos, anotados os seguintes parâmetros: PAS, PAD, FC, BIS, SEF ${ }_{95 \%}$, $\delta \%$, taxa de supressão de surtos, CE e CE/CAM.

RESULTADOS: Os valores de BIS e SEF $F_{95 \%}$ apresentaram correlação direta com a CE/CAM do sevoflurano a valores de $B I S$ de 40, 50, 60 e despertar, respeitando-se a CAM para idade $(p>0,05)$. A $\delta \%$, no $\mathrm{Gl}$, apresentou valores superiores a todos os outros grupos, nos cinco momentos $(p<0,05)$.

CONCLUSÕES: O BIS e SEF ${ }_{95 \%}$ podem ser utilizados na monitorização da profundidade da anestesia com sevoflurano

\footnotetext{
* Recebido do (Received from) Serviço de Anestesiologia do São Lucas Hospital de Cirurgia \& Anestesia. Fortaleza, CE

1. Diretor Clínico e Chefe do Serviço de Anestesiologia do São Lucas - Hospital de Cirurgia \& Anestesia; Mestre em Cirurgia, Área de Concentração: Anestesiologia; Graduando em Engenharia pela Universidade de Fortaleza

2. Coordenadora do Centro de Estudos do São Lucas - Hospital de Cirur gia \& Anestesia; Doutora em Anestesiologia

3. Professor Adjunto da Universidade Federal Fluminense; MSc em Engenharia Biomédica; PhD em Anestesiologia

4. Chefe do Serviço de Anestesiologia do Hospital Luiz de França, Membro do Centro de Estudos do São Lucas- Hospital de Cirurgia \& Anestesia, Fortaleza, CE
}

Endereço para correspondência (Correspondence to)

Dr. Rogean Rodrigues Nunes

Rua Gothardo Moraes, 155/1201, Bloco Dunas - Bairro: Papicu

60190-801 Fortaleza, CE

E-mail: rogean@fortalnet.com.br

Apresentado (Submitted) em 23 de julho de 2002

Aceito (Accepted) para publicação em 22 de outubro de 2002

(C) Sociedade Brasileira de Anestesiologia, 2003 em crianças de 0 a 12 anos observando-se os mesmos parâmetros sugeridos para adultos. O mesmo não acontece com a $\delta \%$, a qual mostrou variações dependentes, provavelmente, da maturação cerebral.

Unitermos: ANESTESIA, Pediátrica; ANESTÉSICOS, Volátil: sevoflurano; MONITORIZAÇÃO: eletroencefalografia, índice bispectral, $\mathrm{SEF}_{95 \%}$, análise espectral da potência

\section{SUMMARY}

Nunes RR, Cavalcante SL, Gemal AE, Amorim DGS - Processed Electroencephalogram in Children Anesthetized with Sevoflurane. Is it Feasible?

BACKGROUND AND OBJECTIVES: EEG-derived bispectral index (BIS), has been indicated as a major substrate for measuring hypnotic effects of anesthetic drugs. However, there are only limited data on the use of EEG in anesthetized children. This study aimed at evaluating changes in $B I S, S E F_{95 \%}$, relative delta band frequency amplitude $(\delta \%)$ and suppression rate $(S R)$ in children, correlating these changes with sevoflurane pharmacodynamic variables (EC and EC/MAC) as compared to adults.

METHODS: Participated in this study 100 patients of both genders, aged 0 to 40 years, physical status ASA I and II. All patients were induced with sevoflurane followed by neuromuscular blocker at BIS 30. Patients were distributed in 5 groups: GI (20) - 0 to 6 months; GIl (20) > 6 months to 2 years; GIII (20) > 2 to 12 years; GIV (20) > 12 to 18 years and $G V(20)>$ 18 to 40 years. Five moments were evaluated for each group: $M_{1}$ (awaken); $M_{2}$ (BIS 60); $M_{3}$ (BIS 50); $M_{4}$ (BIS 40) and $M_{5}$ (emergence). The following parameters were recorded for all moments: $S B P, D B P, H R, B I S, S E F_{95 \%}, \delta \%$, suppression rate, $E C$ and EC/MAC.

RESULTS: Both BIS and SEF $95 \%$ values for all age groups directly correlated to sevoflurane's EC/MAC at BIS values of 40 , 50,60 and at emergence, considering MAC values for age ( $p>$ 0.05 ). $\delta \%$ values in $\mathrm{GI}$ were higher than in any other group during all five moments $(p<0.05)$.

CONCLUSIONS: Unlike $\delta \%$, the variations of which seem brain maturation-related, BIS and SEF ${ }_{95 \%}$ may be used to monitor sevoflurane's anesthetic depth in children aged 0 to 12 years, observing the parameters suggested for adults.

Key Words: ANESTHESIA, Pediatric; ANESTHETICS, Volatile: sevoflurane; MONITORING: electroencephalography, bispectral index, $\mathrm{SEF}_{95 \%}$, power spectral analysis

\section{INTRODUÇÃO}

O dice Bispectral (BIS), um parâmetro derivado do eletroencefalograma (EEG), tem sido indicado como um substrato importante na mensuração do efeito hipnótico de drogas anestésicas no sistema nervoso central ${ }^{1,2}$. Muitos estudos descrevem o uso do índice bispectral como indica- 
dor de profundidade da hipnose durante anestesia inalatória $^{3}$, particularmente em sedações com sevoflurano ${ }^{4,5}$. A utilização do BIS tem sido extensivamente estudada em adultos ${ }^{4-10}$. Entretanto, o uso na população pediátrica ainda é motivo de discussão, pois os dados atualmente disponíveis são limitados e controversos, principalmente em crianças entre 0 e 6 meses de idade ${ }^{11}$. Scher ${ }^{12}$ mostrou que até os seis meses de idade a freqüência dominante no $E E G$ é de 5 $\mathrm{Hz}$; de 9 a 18 meses é 6-7 Hz, aos 2 anos é 7-8 Hz, aos 7 anos é $9 \mathrm{~Hz}$ e aos 15 anos de idade comporta-se como a do adulto: $10 \mathrm{~Hz}$.

Em revisão da literatura, não foram encontrados trabalhos correlacionando agente anestésico único com parâmetros eletroencefalográficos em crianças.

O objetivo deste estudo é avaliar mudanças no BIS, SEF $95 \%$ no canal 1 (esquerdo) e SEF $95 \%$ no canal 2 (direito), amplitude relativa na banda de freqüência delta $(\delta \%)$ - (observar a mudança na maturação da atividade cortical) e taxa de supressão de surtos em crianças, adolescentes e adultos, tanto em fases profundas de hipnose como durante o despertar, correlacionando as variáveis farmacodinâmicas do sevoflurano: concentração expirada (CE) e a relação da CE com a CAM para idade (CE/CAM).

\section{MÉTODO}

Após aprovação pelo Comitê de Ética da instituição, participaram do estudo 100 pacientes (adultos, adolescentes e pediátricos), de ambos os sexos, com idades variando de 0 a 40 anos, estado físico ASA I e II, submetidos a operações para: amigdalectomias, adenoamigdalectomias, neurorrafias, tenorrafias, tratamento de fraturas de membros inferiores e doenças ginecológicas. Foram excluídos do protocolo, pacientes com doenças cardíaca, pulmonar ou neurológica, prematuros, obesos ou aqueles em uso de medicamentos que conhecidamente afetam o eletroencefalograma ou a CAM do sevoflurano. Os dados eletroencefalográficos foram mensurados através de aparelho específico (Aspect A 1000 - versão 3.31$)^{\circledR}$ fabricado para utilização em adultos. Foram utilizados 2 canais referenciais (F7 e F8) com Fz de referência e Fp2 como terra, com eletrodos de prata/cloreto de prata em gel condutor acoplados ao conversor do aparelho, sendo computados os dados após teste de impedância realizado pelo próprio aparelho e posterior liberação, desde que a impedância registrada em todos os canais fosse menor que $5 \mathrm{~K} \Omega$. Os pacientes foram estratificados em 5 grupos de 20, assim dispostos: GI - idade entre zero e 6 meses; Gll: idade maior que 6 meses até 2 anos; GIII: idade maior que 2 anos até 12 anos; GIV: idade maior que 12 anos até 18 anos; GV: idade maior que 18 anos até 40 anos. Em nenhum dos pacientes foi utilizada medicação pré-anestésica, sendo solicitada a presença da mãe na sala de operação nos grupos I, II e III durante a indução da anestesia.

$\mathrm{Na}$ sala de operação, todos os pacientes foram monitorizados com: esfigmomanômetro para aferição das pressões ar- teriais sistólica (PAS) e diastólica (PAD), através de método automático, freqüência cardíaca (FC), oxímetro de pulso para medida da $\mathrm{SpO}_{2}$, capnógrafo para medida da $\mathrm{P}_{\mathrm{ET}} \mathrm{CO}_{2}$, analisador de agentes anestésicos, cardioscopia em $D_{\|} e$ V5, índice bispectral (BIS), freqüência de borda espectral 95\% $\left(\mathrm{SEF}_{95 \%}\right)$ em dois canais, amplitude relativa na banda de freqüência delta em dois canais $(\delta \%)$, taxa de supressão de surtos (TS) e monitorização do bloqueio neuromuscular através da seqüência de quatro estímulos (SQE) a cada 14 segundos, utilizando o adutor do polegar (aceleromiografia), após detecção do estímulo supramaximal, imediatamente antes da injeção do mivacúrio. Nos grupos I, II e III a punção venosa foi realizada após indução anestésica. Todos os pacientes foram induzidos com sevoflurano e óxido nitroso a $60 \%$ em oxigênio, através da técnica do volume corrente, até um BIS de 30 , momento em que foram administrados $80 \mu \mathrm{g} \cdot \mathrm{kg}^{-1}$ de mivacúrio por via venosa, para facilitar a inserção da máscara laríngea (realizada após ser obtido 5\% T1 na SQE) e posterior ventilação mecânica com ventilador com compensação de perda de volume, seguido de exclusão do óxido nitroso. A ventilação mecânica foi ajustada em todos os pacientes para gerar um volume corrente equivalente a $8 \mathrm{ml} . \mathrm{kg}^{-1}$ e freqüência respiratória adaptada para manter $\mathrm{P}_{\mathrm{ET}} \mathrm{CO}_{2}$ entre 35 e $45 \mathrm{mmHg}$. Foi utilizado sistema circular com reabsorvedor de $\mathrm{CO}_{2}$, com fluxo de $1 \mathrm{~L}$. $\mathrm{min}^{-1}$ de $\mathrm{O}_{2}$ e sevoflurano vaporizado através de equipamento específico. Para fins de estudos clínico e estatístico foram analisados 5 momentos $M_{1}$ : antes da indução anestésica; $M_{2}$ : BIS de $60 ; M_{3}$ : BIS de 50; $\mathrm{M}_{4}$ : BIS de 40 e $\mathrm{M}_{5}$ : BIS ao despertar, aqui considerado quando o paciente abre os olhos ou esboça algum movimento coordenado.

A anestesia geral utilizada para possibilitar a realização dos procedimentos cirúrgicos citados iniciou-se logo após o $\mathrm{M}_{5}$, em todos os grupos, evitando-se, assim, a influência dos diversos estímulos cirúrgicos no nível de hipnose, nos momentos estudados. Para cada ajuste no valor do BIS, exceto $M_{1}$, foi padronizado o tempo de 5 minutos e o valor da CE do sevoflurano foi anotado após 10 minutos de estabilização nos referidos valores de BIS (40,50 e 60). Em cada momento citado foram anotados, também, os seguintes parâmetros: PAS, PAD, FC, BIS, SEF $95 \%$ nos canais 1 e $2, \delta \%$, TS, CE, $\mathrm{SpO}_{2}$ e SQE. Variações de $\pm 25 \%$ para PAS, PAD e FC, em relação aos valores de $\mathrm{M}_{1}$, foram consideradas sem significância clínica, neste trabalho.

Em todos os pacientes, a temperatura nasofaríngea foi mantida entre 36 e $37^{\circ} \mathrm{C}$, com auxílio de lençol de ar térmico forçado convectivo.

Para verificar se existiam diferenças significativas entre as médias das variáveis consideradas, utilizou-se a Análise de Variância com medidas repetidas, seguidas do teste de Tukey quando necessário, sendo este aplicado para as médias das variáveis em relação aos grupos dentro de cada momento e para as médias das variáveis em relação aos momentos dentro de cada grupo, considerando-se como significativos os valores de $p<0,05$. 


\section{RESULTADOS}

Os dados demográficos estão apresentados na tabela I. Os valores médios nas variáveis PAS, PAD e FC, relacionando momento dentro de cada grupo podem ser vistos na tabela II, figuras 1, 2 e 3 . A análise destes dados mostra que em todos os grupos estudados da variável PAS, o momento $\mathrm{M}_{3}$ diferiu significativamente do $M_{1}(p<0,05)$. Entretanto, excetuando-se os grupos IV e $\mathrm{V}$, todos os outros valores no $\mathrm{M}_{1}$ foram semelhantes ao $\mathrm{M}_{5}(p>0,05)$. Em relação a PAD, apesar de não ultrapassarem valores referenciais para as faixas etárias, os momentos $\mathrm{M}_{2}, \mathrm{M}_{3}$ e $\mathrm{M}_{4}$ diferiram significativamente dos momentos $\mathrm{M}_{1}$ e $\mathrm{M}_{5}$, em todos os grupos, sendo o $\mathrm{M}_{5}$ significativamente diferente do $M_{1}$ apenas no grupo III. Na variável FC, apenas o grupo I apresentou variações significativas entre todos os momentos, sendo o grupo II diferente apenas nos momentos $\mathrm{M}_{1}, \mathrm{M}_{4}$ e $\mathrm{M}_{5}(\mathrm{p}<0,05)$ e os grupos: III, IV e V, diferentes nos momentos $M_{2}, M_{3}$ e $M_{5}(p<0,05)$ sem, entretanto, mostrarem variações clinicamente significativas. A análise do BIS por grupo, dentro de cada momento, evidenciou que nos momentos $M_{1}, M_{2}, M_{3}$ e $M_{4}$, os grupos não apresentaram variações estatisticamente significativas $(p>0,05)$. No momento $\mathrm{M}_{5}$ os grupos I e IV evidenciaram valores seme-
Ihantes, mas significativamente diferentes dos grupos II, III e $V$ sem, contudo, extrapolarem valores relacionados ao despertar (Tabela III e Figura 4).

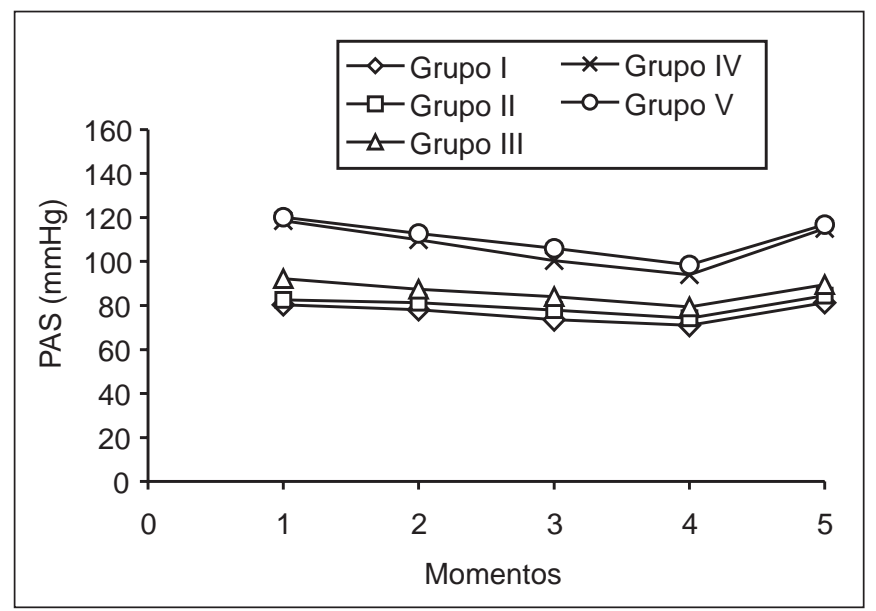

Figura 1 - Variações na Pressão Arterial Sistólica (PAS) $(\mathrm{mmHg})$, por Momento dentro de Cada Grupo

$\left(p>0,05\right.$ para $M_{1}$ e $M_{5}$, exceto nos grupos IV e V). Análise pelo teste de Tukey

Tabela I - Dados Demográficos

\begin{tabular}{|c|c|c|c|c|c|}
\hline \multirow[t]{2}{*}{ Variáveis } & \multicolumn{5}{|c|}{ Grupos } \\
\hline & 1 & II & III & IV & V \\
\hline Idade (anos) * & $0,30 \pm 0,14$ & $1,26 \pm 0,51$ & $7,36 \pm 3,00$ & $15,3 \pm 1,74$ & $27,2 \pm 7,19$ \\
\hline Peso $(\mathrm{kg})$ * & $5,37 \pm 1,06$ & $10,16 \pm 2,08$ & $24,32 \pm 7,43$ & $51,2 \pm 9,61$ & $65,75 \pm 4,89$ \\
\hline \multicolumn{6}{|l|}{ Estado físico } \\
\hline ASA I & 17 & 15 & 15 & 10 & 14 \\
\hline ASA II & 03 & 05 & 05 & 10 & 06 \\
\hline \multicolumn{6}{|l|}{ Sexo } \\
\hline Masculino & 13 & 12 & 18 & 11 & 12 \\
\hline Feminino & 07 & 08 & 02 & 09 & 08 \\
\hline
\end{tabular}

*Valores expressos pela Média \pm DP

Tabela II - Pressão Arterial Sistólica (PAS), Pressão Arterial Diastólica (PAD)-(mmHg) e Freqüência Cardíaca (FC) (bat. $\mathrm{min}^{-1}$ ), por Grupo dentro de Cada Momento, (Média \pm DP)

\begin{tabular}{|c|c|c|c|c|c|c|}
\hline \multirow[t]{2}{*}{ Momentos } & \multirow[t]{2}{*}{ Variáveis } & \multicolumn{5}{|c|}{ Grupos } \\
\hline & & 1 & II & III & IV & $\mathrm{V}$ \\
\hline \multirow[t]{3}{*}{$\overline{M_{1}}$} & PAS & $80,30 \pm 1,30$ & $82,55 \pm 1,85$ & $92,20 \pm 11,33$ & $118,45 \pm 5,26$ & $120,10 \pm 6,99$ \\
\hline & PAD & $50,85 \pm 1,98$ & $61,95 \pm 1,36$ & $62,95 \pm 3,30$ & $74,45 \pm 4,39$ & $76,85 \pm 5,76$ \\
\hline & FC & $130,40 \pm 5,53$ & $121,70 \pm 4,50$ & $106,65 \pm 12,31$ & $82,60 \pm 5,29$ & $79,30 \pm 3,99$ \\
\hline \multirow[t]{2}{*}{$\mathrm{M}_{2}$} & PAS & $78,05 \pm 3,28$ & $81,15 \pm 2,66$ & $87,30 \pm 7,85$ & $109,90 \pm 3,88$ & $112,75 \pm 6,76$ \\
\hline & PAD & $45,70 \pm 2,25$ & $59,30 \pm 2,32$ & $61,65 \pm 2,23$ & $68,80 \pm 4,82$ & $67,70 \pm 3,20$ \\
\hline \multirow[t]{3}{*}{$M_{3}$} & PAS & $73,55 \pm 3,12$ & $77,85 \pm 2,11$ & $83,90 \pm 6,83$ & $100,30 \pm 2,85$ & $105,95 \pm 7,05$ \\
\hline & PAD & $46,40 \pm 2,46$ & $57,50 \pm 1,70$ & $58,20 \pm 3,74$ & $67,60 \pm 2,66$ & $64,00 \pm 2,71$ \\
\hline & $\mathrm{FC}$ & $120,20 \pm 7,42$ & $112,20 \pm 4,64$ & $95,70 \pm 13,08$ & $72,80 \pm 6,67$ & $67,50 \pm 5,37$ \\
\hline \multirow[t]{3}{*}{$\mathrm{M}_{4}$} & PAS & $70,95 \pm 3,07$ & $74,10 \pm 2,81$ & $79,30 \pm 5,87$ & $93,85 \pm 3,23$ & $98,45 \pm 1,99$ \\
\hline & PAD & $42,20 \pm 3,96$ & $52,00 \pm 4,09$ & $54,85 \pm 3,03$ & $64,30 \pm 3,87$ & $60,00 \pm 1,89$ \\
\hline & $\mathrm{FC}$ & $116,85 \pm 6,63$ & $105,30 \pm 4,53$ & $87,70 \pm 11,57$ & $66,40 \pm 6,64$ & $63,45 \pm 4,64$ \\
\hline \multirow[t]{2}{*}{$\mathrm{M}_{5}$} & PAS & $133,65 \pm 3,80$ & $64,50 \pm 1,50$ & $89,45 \pm 9,71$ & $114,95 \pm 2,82$ & $116,70 \pm 6,42$ \\
\hline & $\mathrm{FC}$ & $133,65 \pm 3,80$ & $126,70 \pm 2,70$ & $109,60 \pm 12,71$ & $85,35 \pm 6,91$ & $79,90 \pm 5,48$ \\
\hline
\end{tabular}




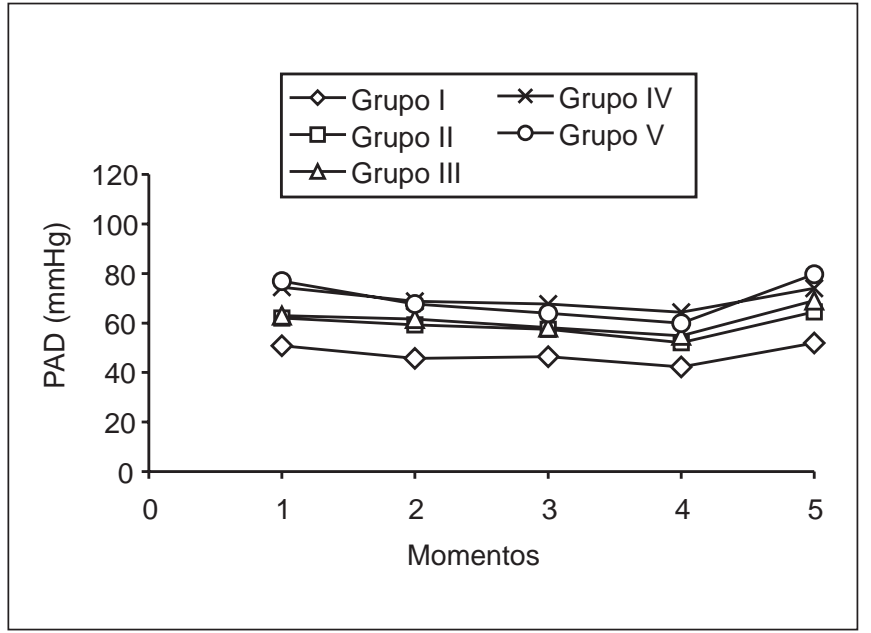

Figura 2 - Variações na Pressão Arterial Diastólica (PAD) (mmHg), por Momento dentro de Cada Grupo

Os momentos $M_{2}, M_{3}$ e $M_{4}$ diferiram significativamente dos momentos $M_{1}$ e $M_{5}$ em todos os grupos - $(p<0,05)$. Análise pelo teste de Tukey

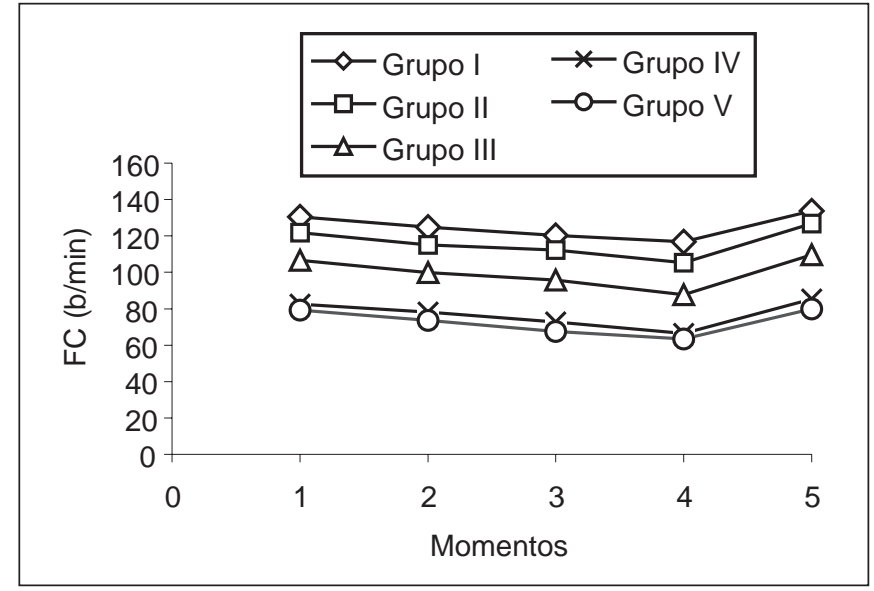

Figura 3 - Variações na Freqüência Cardíaca $(F C)(\mathrm{mmHg})$, por Momento dentro de Cada Grupo

$(p<0,05$ entre todos os momentos no grupo $I ; p>0,05$ para os momentos $M_{1}$ e $M_{5}$ nos grupos III, IV e V). Análise pelo teste de Tukey

Tabela III - Variáveis: BIS, SEF $95 \% 1(\mathrm{~Hz}), \mathrm{SE}_{95 \%} 2(\mathrm{~Hz}), \delta \%, \mathrm{CE}(\%)$ e CE/CAM por Grupo dentro de Cada Momento, (Média \pm DP)

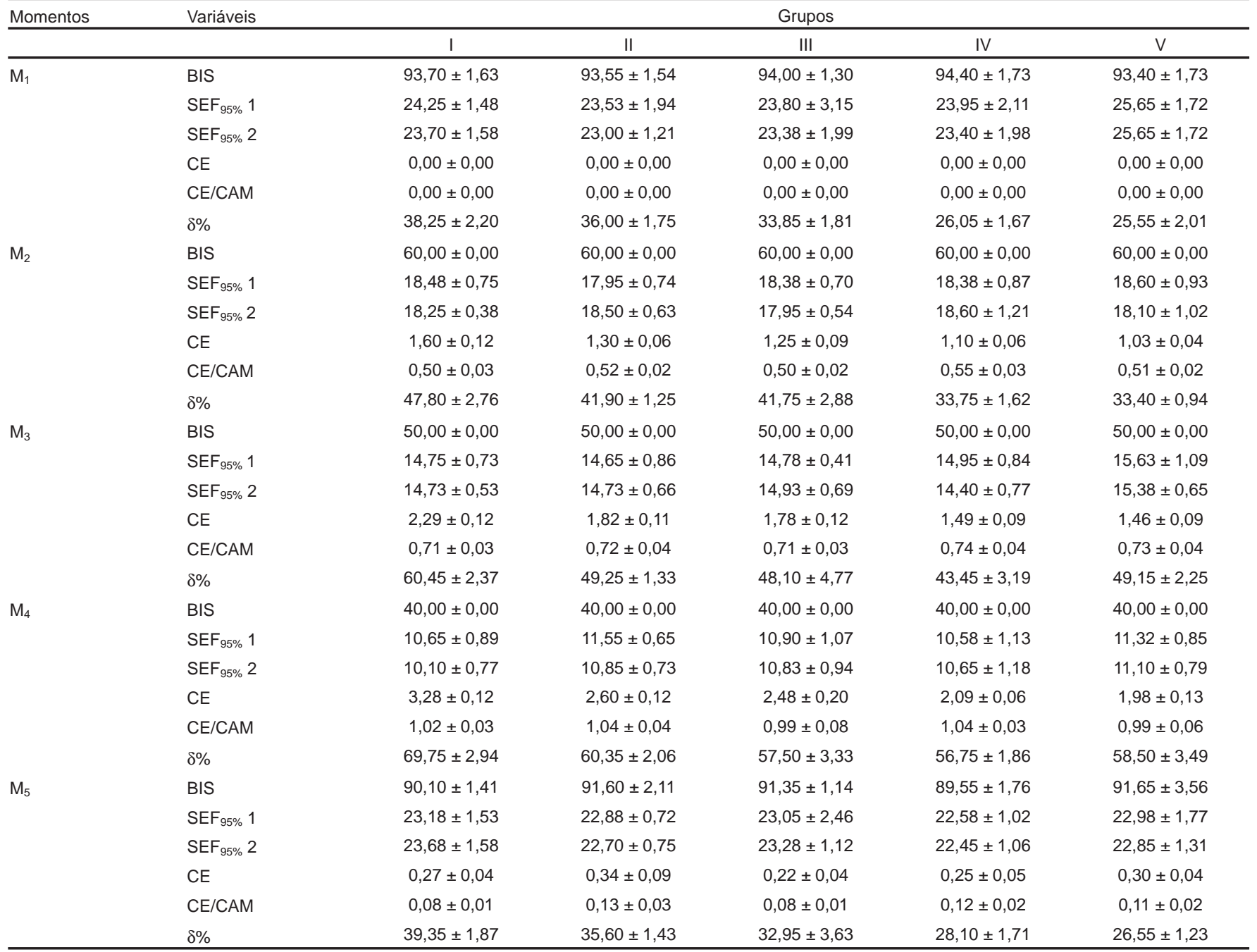




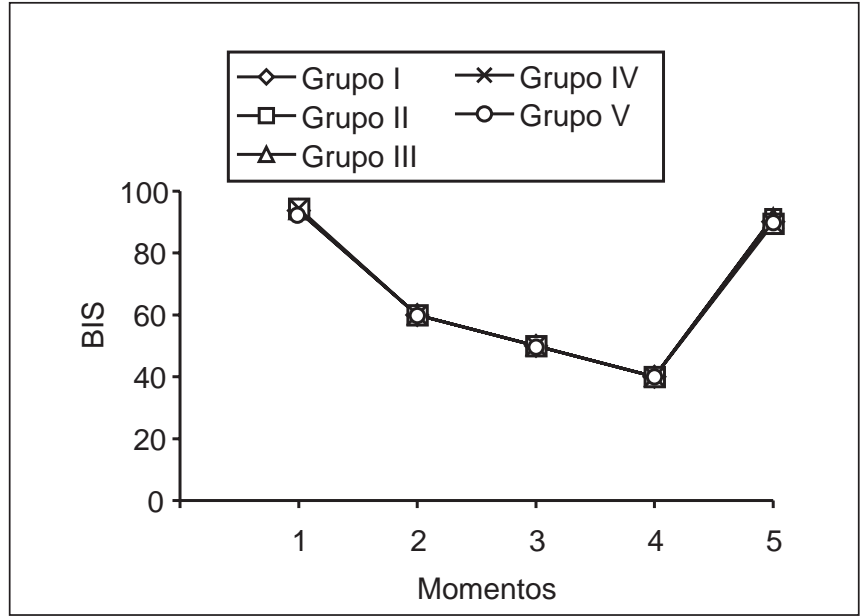

Figura 4 - Variações no Índice Bispectral (BIS)

$p>0,05$ nos momentos $M_{1}, M_{2}, M_{3}$ e $M_{4}$, analisando todos os grupos em cada momento. Análise pelo teste de Tukey

A análise espectral da potência, através do $\mathrm{SEF}_{95 \%}$ nos canais 1 e 2 , relativo ao momento dentro de cada grupo, mostrou que apenas os momentos $\mathrm{M}_{1}$ e $\mathrm{M}_{5}$, em todos os grupos, apresentaram resultados estatisticamente semelhantes $(p>0,05)$ (Tabela III, Figuras 5 e 6 ).

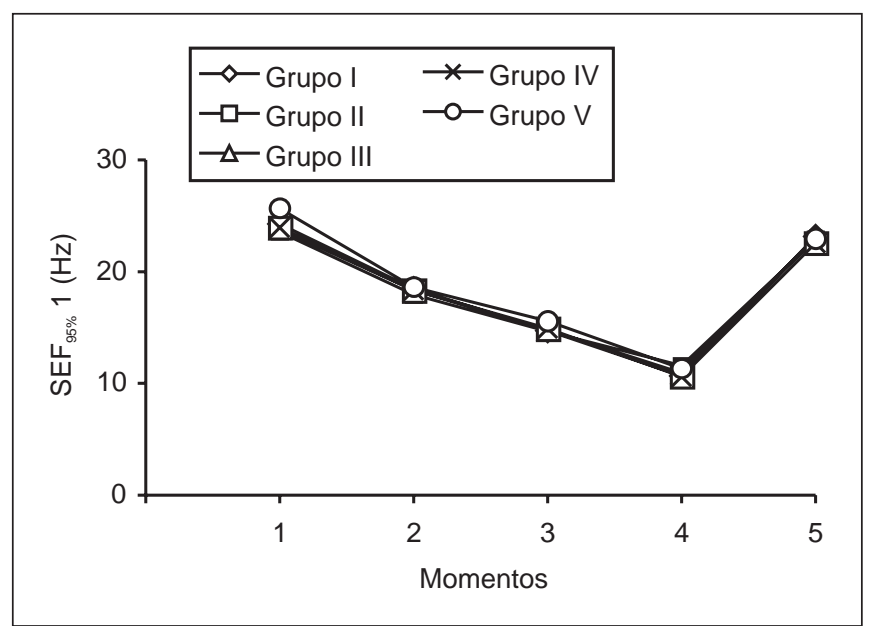

Figura 5 - Variações no $\mathrm{SEF}_{95 \%} 1(\mathrm{~Hz})$

$p>0,05$ nos momentos $M_{1}$ e $M_{5}$, dentro de cada grupo. Análise pelo teste de Tukey

A análise percentual da banda de freqüência delta, resultante dos canais 1 e 2 , mostrou que nos momentos estudados, os valores encontrados no grupo I foram estatisticamente diferentes de todos os outros grupos, registrando, sempre, os maiores valores $(p<0,05)$ (Tabela III e Figura 7). A análise da concentração expirada, observando-se os grupos dentro de cada momento, mostrou que nos momentos $\mathrm{M}_{2}, \mathrm{M}_{3}$ e $\mathrm{M}_{4}$ os grupo II e III apresentaram resultados semelhantes $(p>0,05)$, mas diferentes dos observados entre os grupos IV e V (estes semelhantes entre si).

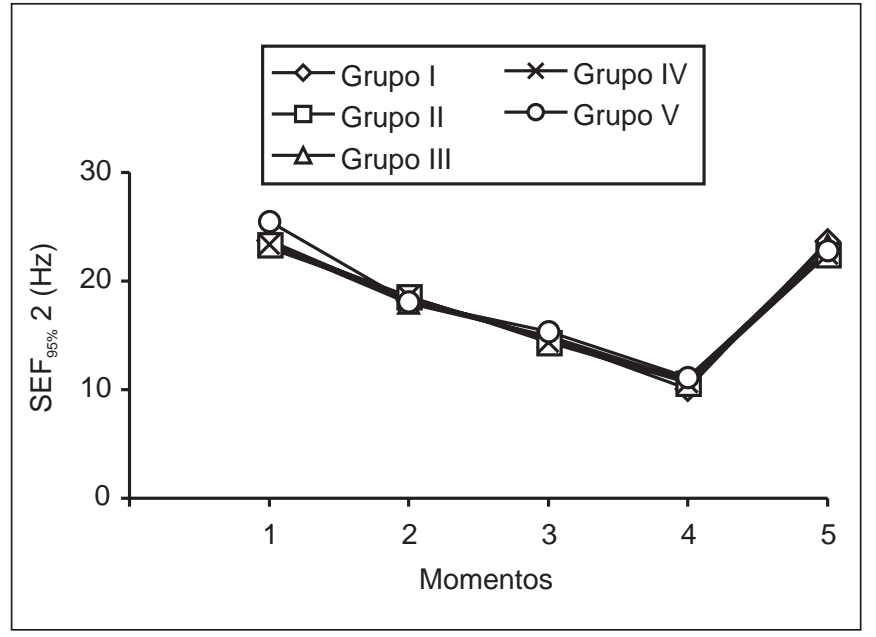

Figura 6 - Variações no $\mathrm{SEF}_{95 \%} 2(\mathrm{~Hz})$ $p>0,05$ nos momentos $M_{1} \in M_{5}$, dentro de cada grupo. Análise pelo teste de Tukey

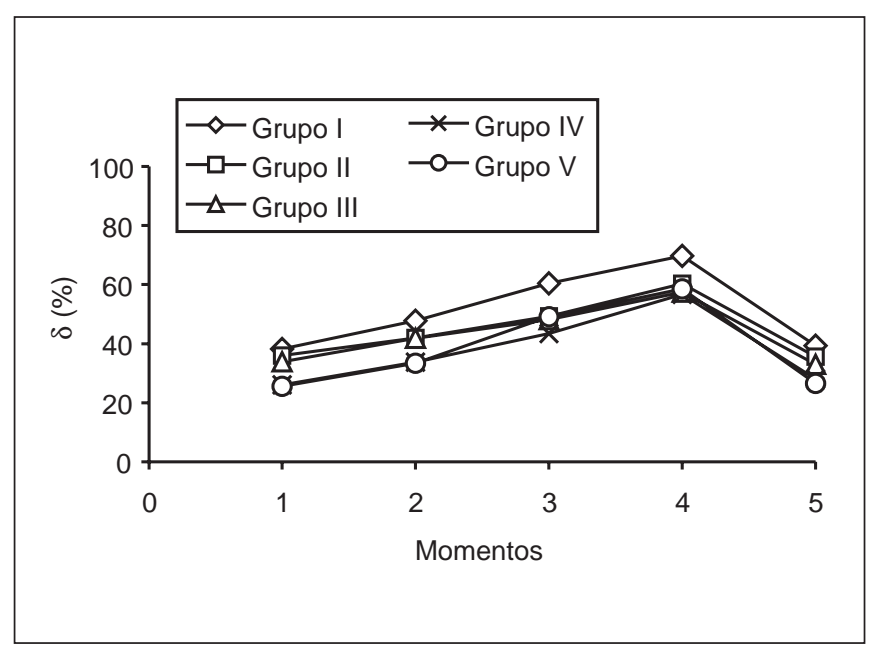

Figura 7 - Variações no $\delta \%$

$\mathrm{p}<0,05$ nos momentos $M_{1}, M_{2}, M_{3}, M_{4}$ e $M_{5}$, dentro de cada grupo. Análise pelo teste de Tukey

Entretanto, os valores observados nos momentos $M_{2}, M_{3}$ e $\mathrm{M}_{4}$ para o grupo I foram estatisticamente diferentes de todos os outros grupos, tendo estes momentos mostrado os maiores escores $(p>0,05)$. Para o $M_{5}$, todos os valores encontrados para os grupos estudados diferiram significativamente $(p<0,05)$. Contudo, os valores médios observados mantiveram variação entre $0,27 \%$ e $0,34 \%$. Os valores dos dados farmacocinéticos (CE e CE/CAM) e BIS estão ilustrados na tabela III e figuras 8 e 9 . Na análise dos grupos dentro de cada momento para a variável CE/CAM, não foram observadas diferenças significativas em $M_{2}, M_{3}$ e $M_{4}$ $(p>0,05)$. Já os valores encontrados em $M_{5}$ (despertar) mostraram semelhança estatística entre os grupos II, IV e $V$, os quais diferiram significativamente $(p<0,05)$ dos grupos I e III (estes semelhantes entre si). Entretanto, apesar 


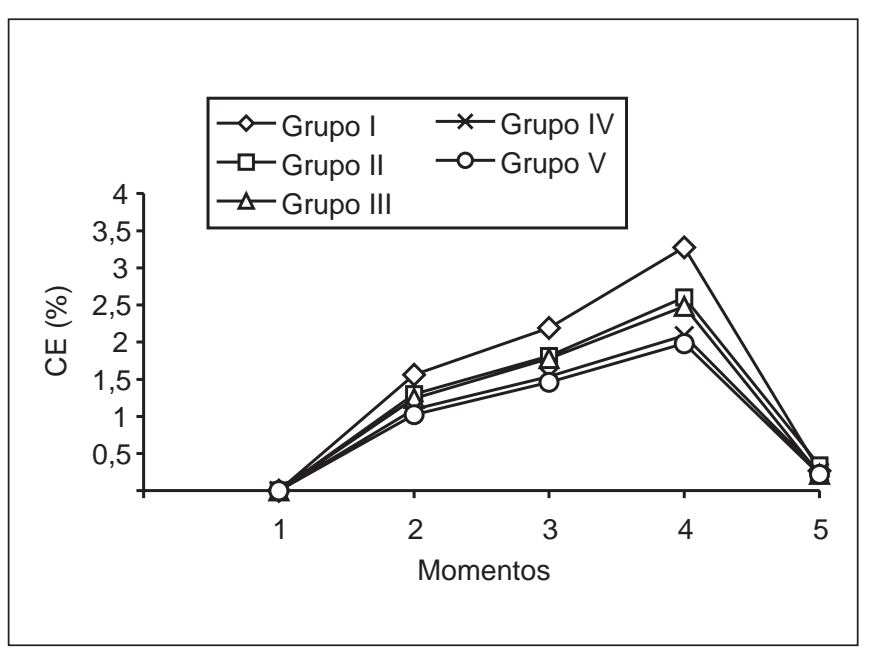

Figura 8 - Variações da Concentração Expirada (CE)

$\mathrm{Na}$ análise dos grupos dentro de cada momento observamos: $p>0,05$ para $M_{2}, M_{3}$ e $M_{4}$ nos grupos II e III. $p>0,05$ para $M_{2}$, $\mathrm{M}_{3}$ e $\mathrm{M}_{4}$ nos grupos IV e V, com $\mathrm{p}<0,05$ comparando-se os grupos II e III aos grupos IV e V nestes mesmos momentos. $\mathrm{p}<$ 0,05 para $M_{2}, M_{3}$ e $M_{4}$ no grupo I comparando-se a todos os outros grupos

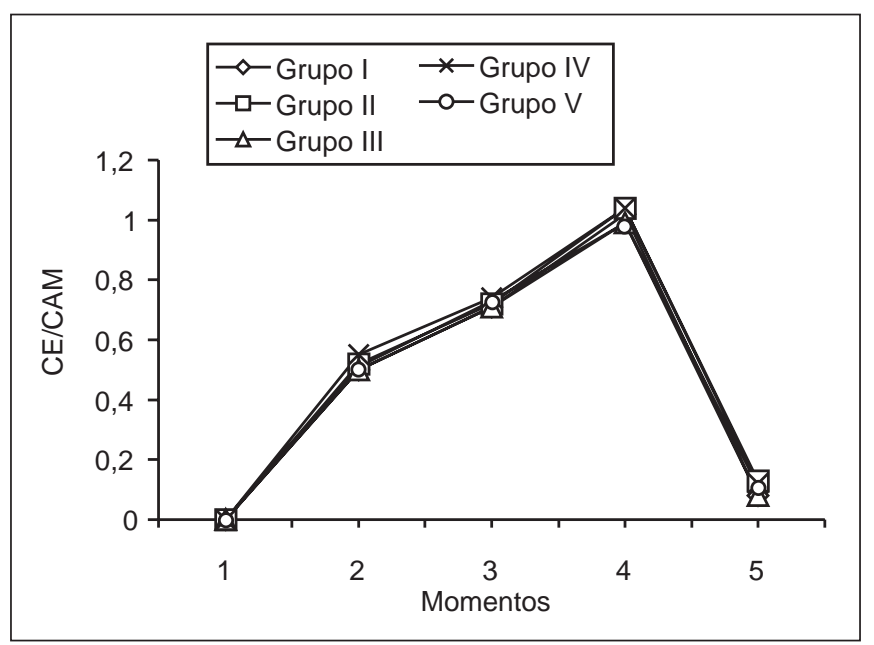

Figura 9 - Variações na CE/CAM

Análise dos grupos dentro de cada momento. $p>0,05$ em $\mathrm{M}_{2}$, $\mathrm{M}_{3}$ e $\mathrm{M}_{4}$

de o maior valor observado da relação CE/CAM ser de 0,13 (Grupo II em $\mathrm{M}_{5}$ ), este representa apenas $43 \%$ da CAM acordada (0,3 CAM) para o sevoflurano ${ }^{13}$. A TS não apresentou valores diferentes de zero, nos momentos estudados. Antes do despertar, a SQE já indicava valores maiores que 0,9 em todos os pacientes.

\section{DISCUSSÃO}

Não se conhece como os dados fornecidos pelo eletroencefalograma processado (BIS e $\mathrm{SEF}_{95 \%}$ ) possam ser diferentes em crianças quando comparados com adultos, particular- mente em crianças com menos de 6 meses de idade e, possivelmente, até os cinco anos de idade, pois a maturação do cérebro e formação de sinapses continuam mesmo depois do nascimento e vão até os 5 anos, com a maioria destas alterações ocorrendo em momentos mais cedo da vida ${ }^{14}$. Porém, Denmam e col., em trabalho recente avaliando crianças entre 0 e 12 anos de idade ${ }^{15}$, indicaram que a relação dose resposta sevoflurano/BIS é similar em crianças respirando sevoflurano/ $\mathrm{N}_{2} \mathrm{O}$ àquela vista em adultos respirando sevoflurano $/ \mathrm{O}_{2}{ }^{8}$, sendo a concentração de sevoflurano para BIS 50 de $1,55 \%$ para crianças menores que 2 anos de idade e para crianças entre 2 e 12 anos de idade de 1,25\%. Entretanto, neste estudo, Denman reconhece que a utilização de medicação pré-anestésica produziu substancial efeito sedativo, o que refletiu em diminuições nos valores do BIS. Apesar desta consideração, o estudo sugere que o mesmo equipamento e método de análises eletroencefalográficas podem ser aplicados em crianças. Entretanto, Bannister e col. ${ }^{11}$ avaliando o efeito da monitorização do índice bispectral na anestesia e durante o período de recuperação da anestesia em crianças, utilizando sevoflurano associado ao óxido nitroso, mostraram que existe dificuldade em ajustar concentrações de sevoflurano para alcançar um valor de BIS pré-determinado no grupo entre 0 e 6 meses de idade que foram submetidas a bloqueio peridural caudal. Além disso, neste mesmo trabalho, os valores de BIS permaneceram baixos apesar da descontinuidade precoce do sevoflurano, o que não aconteceu no grupo de pacientes mais velhos. Uma das explicações dos autores seria, talvez, devida às diferenças eletroencefalográficas nesta população, determinadas pela maturação e formação de sinapses que ocorrem nos primeiros meses de vida ${ }^{16-18}$. Outra explicação seria a presença de óxido nitroso a $60 \%$, o qual, sozinho, poderia ser suficiente para produzir um estado de hipnose profunda nesta população, concluindo que mais dados seriam necessários para interpretação do BIS nesta faixa etária. Entretanto, Bannister e col. também introduziram na técnica um fator potencialmente confuso na comparação da relação BIS/idade: os pacientes mais jovens e submetidos à correção de hérnia inguinal foram submetidos a bloqueio caudal, o que não ocorreu com os mais velhos, pois estes foram operados de amígdalas e/ou adenóides. $O$ fator é potencialmente confuso, pois estudos recentes mostram uma redução na necessidade de sedação per-operatória em adultos após bloqueio regional ${ }^{19,20}$. Além disso, os autores não referiram os valores de BIS no momento do despertar, nesta população. Davidson e col. ${ }^{21}$ em recente estudo avaliando as diferenças no índice bispectral em crianças durante o despertar da anestesia após operações de fimose (53 pacientes) com menos de 15 anos de idade, sendo todos induzidos com sevoflurano e óxido nitroso em oxigênio, sob máscara, mostraram que o BIS imediatamente antes do despertar tende a ser mais baixo em crianças entre 5 e 11 meses de idade $(67,8 \pm 10)$ quando comparado a crianças entre $12 \mathrm{e}$ 178 meses de idade $(73,5 \pm 7)$ e que após o despertar, os valores médios de BIS foram: crianças entre 5 e 11 meses de idade $(85,6 \pm 13,6)$ e crianças entre 12 e 178 meses de idade 
$(83,1 \pm 12)$, concluindo que o BIS pode ser aplicado em crianças da mesma forma que em adultos, mas, sua validação no grupo de 5 a 11 meses merece mais estudos. Entretanto, os autores investigaram os valores de BIS a baixas concentrações de sevoflurano, ou seja, planos superficiais de anestesia, sem demonstrar uma relação clara entre BIS e concentração expirada do agente anestésico, o qual foi fixado em 0,9\%, 0,7\% e 0,5\%, sem levar em consideração as variações de CAM nas diversas faixas etárias ${ }^{22}$, o que pode resultar em variações importantes nos valores de BIS e SEF ${ }_{95 \%}$. Glass e col. ${ }^{4}$ demonstraram perda da consciência com BIS médio de $71 \mathrm{em}$ pacientes anestesiados com isoflurano. Katot e col. ${ }^{8}$ mostraram que o valor de BIS em que adultos sedados com sevoflurano responderam a um comando em voz alta foi de 73. Assim, Davidson e col. mostraram que o BIS em crianças menores (5 a 11 meses) deve ser interpretado com cautela; pois, embora haja um significativo aumento no BIS neste grupo após despertar estimulado, estes têm um baixo BIS comparado com crianças, maiores (12 a 178 meses), imediatamente antes do despertar, além de apresentarem uma larga variação nos valores do BIS, não sendo demonstrada, também, nenhuma correlação entre a concentração expirada de sevoflurano e BIS durante o despertar, o que contrasta com recente estudo de Denman e col. ${ }^{15}$. Além disso, este estudo de Davidson e col. manteve ventilação sob máscara durante a operação, o que dificulta a regularidade da freqüência respiratória e volume corrente, assim como uma manutenção de equilíbrio mais adequado nos valores da concentração expirada de sevoflurano.

Degout e col. ${ }^{23}$, em recente trabalho avaliando BIS e componentes hipnóticos da anestesia induzida por sevoflurano e comparando crianças entre 3,5 e 13 anos com adultos entre 22 e 72 anos, mostraram que o BIS mantém boa correlação com componentes hipnóticos da anestesia em crianças e adultos induzidos por sevoflurano. Entretanto, neste mesmo estudo, os autores utilizaram, em todos os pacientes, sedativos como medicação pré-anestésica, além de bolus de alfentanil antes da intubação traqueal e manutenção em infusão contínua durante a operação, o que altera diretamente a avaliação da correlação entre sevoflurano e BIS.

Johansen e col. ${ }^{24}$ em trabalho avaliando continuamente o pré-operatório com BIS e resultados pós-operatórios em crianças entre 2 e 12 anos, mostraram que um BIS de 85 representa um adequado despertar para extubação traqueal e que o BIS pode ser um instrumento útil na anestesia pediátrica.

O presente estudo avaliou a correlação de parâmetros farmacodinâmicos com derivados do eletroencefalograma, utilizando o sevoflurano, um agente inalatório que apresenta baixo coeficiente de partição sangue/gás, não pungente, não inflamável e com limitada depressão cardiorrespiratória, podendo ser útil para uso em adultos e crianças ${ }^{25-27}$. Prévios estudos com halotano, isoflurano e desflurano mostram que a CAM aumenta com diminuição da idade. A farmacologia do sevoflurano em neonatos e crianças foi bem determinada em estudo realizado por Lerman e col. ${ }^{22}$, sendo demonstrado que a CAM média em neonatos é de 3,2\%; em crianças com mais de 1 e até 6 meses, de 3,2\%; em crianças com mais de 6 até 12 meses, de 3,2\% e crianças com mais de 1 até 12 anos, de $2,5 \%$. O valor da CAM do sevoflurano em adultos é de $2 \%$ 13

A correlação entre os diversos grupos/momentos, no presente estudo foi: Grupo I ( 0 a 6 meses): $M_{1}$ (acordado) evidenciou que os valores médios de BIS são compatíveis com pacientes adultos despertos e conscientes. Além disso, os valores do $\mathrm{SEF}_{95 \%}$ nos dois canais estudados também se mostraram correlacionar com pacientes despertos. Entretanto, na avaliação do percentual de densidade espectral $(0-30 \mathrm{~Hz})$ na banda de freqüência delta foi observado que o mesmo apresentou valores elevados em relação a de adultos, o que está de acordo com trabalho Scher ${ }^{12}$ em que o autor afirma que nesta faixa etária a freqüência predominante em pacientes despertos localiza-se entre $0-5 \mathrm{~Hz}$, o que não influenciou os valores de borda espectral e nem o BIS, comparativamente a adultos. $\mathrm{Em} \mathrm{M}_{2}$ (BIS 60), os pacientes não mais responderam a estímulos táteis ou verbais. Foi observada redução no SEF $_{95 \%}$ nos dois canais assim como aumento no percentual de densidade espectral nas freqüências de banda delta, o que é compatível com aprofundamento da anestesia. Estas alterações ocorreram com valor médio de CE do sevoflurano correspondente a 1,6\% e CE/CAM médio igual a $0,5 . M_{3}$ (BIS 50) foi observado maior redução no $\mathrm{SEF}_{95 \%}$ (dois canais), assim como maior aumento no $\delta \%$ e CE média correspondente a 2,29\% e CE/CAM médio de 0,71 , mostrando maior aprofundamento da anestesia. $\mathrm{M}_{4}$ (BIS 40): neste momento foram observados os menores valores de $\mathrm{SEF}_{95 \%}$, assim como os maiores valores de $\delta \%$, o que continua compatível com maior profundidade da anestesia (lentificação da atividade elétrica, com aumento da potência espectral em faixas de freqüências baixas). O valor médio da CE foi de $3,28 \%$ e da CE/CAM de 1,02. M 5 (despertar) - neste momento, foi observada a regressão dos parâmetros eletroencefalográficos ( $\mathrm{SEF}_{95 \%}$, BIS e $\delta \%$ ), os quais retornaram a valores próximos aos basais e correspondentes a clínica. Não avaliamos, durante o despertar, o tempo correspondente ao mesmo, pois acreditamos que o mais importante seria a análise da correlação entre os valores do EEG e os dados clinicamente pré-estabelecidos para o despertar, avaliação esta que se mostrou confusa em trabalho de Bannister e col. ${ }^{11} \mathrm{em}$ pacientes entre 0 a 6 meses de idade submetidos à herniorrafia inguinal, em que o mesmo utilizou anestesia geral/regional, além da utilização de $\mathrm{N}_{2} \mathrm{O}$, mostrando que a despeito de reduções importantes, durante as operações, na administração de sevoflurano, os valores de BIS permaneceram abaixo de 40. Entretanto, não foi claro na avaliação da correlação clínica/EEG, além de sugerir que o óxido nitroso a $60 \%$ sozinho poderia ser suficiente para manter um profundo estado de hipnose nesta população de crianças.

No grupo Il (6 meses a 2 anos), em $\mathrm{M}_{1}$ (acordado), os valores de BIS e SEF $95 \%$ nos canais 1 e 2 também foram semelhantes aos de pacientes adultos (despertos). Em relação aos momentos $M_{2}, M_{3}, M_{4}$ e $M_{5}$, as variações relacionadas ao BIS e 
SEF $_{95 \%}$ comportaram-se de maneira semelhante aos grupos I, III, IV e V. Entretanto, os valores absolutos da CE, momento a momento, foram aumentando à medida que diminuíam os valores de BIS e SEF ${ }_{95 \%}$, mas os valores da CE relativamente a CAM (CE/CAM) para esta faixa etária permaneciam semeIhantes ao grupo I, momento a momento. Na avaliação da $\delta \%$, em todos os momentos foram observadas reduções nas densidades espectrais, o que pode estar relacionada com a evolução da maturação cerebral. Os dados eletroencefalográficos (BIS, SEF $95 \%$ e $\delta \%$ ) retornaram a valores próximos aos basais $\left(M_{1}\right)$ no momento $M_{5}$.

No grupo III (2 a 12 anos), a análise das variáveis EEG (BISe $\mathrm{SEF}_{95 \%}$ ), assim como a CE e CE/CAM, em todos os momentos, foram semelhantes ao do grupo II. Entretanto, continuouse observando uma redução de densidade espectral média na banda de freqüência delta em todos os momentos quando comparado ao grupo II.

Os grupos IV (12 a 18 anos) e V (18 a 40 anos) apresentaram comportamentos semelhantes em todos os momentos para as variáveis (BIS, SEF $_{95 \%}$ ), CE e CE/CAM, sendo demonstrado, ainda, que para valores de BIS 40, a CE de sevoflurano nesses dois grupos foi semelhante a 1 CAM, relação que se manteve em todos os grupos estudados em valores de BIS 40. Além disso, foi observado que o $\delta \%$ nestes dois grupos comportou-se de maneira semelhante em todos os momentos, sendo observado no $\mathrm{M}_{1}$ que suas densidades espectrais eram percentualmente menores que nos grupos anteriores, configurando deslocamento de potência espectral para freqüências maiores neste momento. A análise global deste estudo mostra que houve mudanças importantes nas densidades espectrais em relação a $\delta \%$, havendo redução da mesma à medida que se observa aumento da idade em pacientes acordados em todos os grupos. Entretanto, as variações espectrais durante anestesia mostram comportamento semeIhante em todos os grupos, ou seja, com paciente anestesiado ocorreu aumento da densidade espectral na banda de freqüências delta $(0-5 \mathrm{~Hz})$, sendo os maiores valores absolutos registrados no grupo I. Já os valores de $S E F_{95 \%}$ nos canais 1 e 2, comportaram-se de maneira semelhante entre adultos, adolescentes e crianças em todos os momentos. Os valores de BIS pré-determinados ou não, relacionados aos dados farmacodinâmicos do sevoflurano, mostraram relação direta com múltiplos da CAM (CE/CAM) nas diversas faixas etárias em todos os momentos, sendo a validação destes dados realizada sem influências de: medicação pré-anestésica, óxido nitroso, estímulos cirúrgicos, bloqueios anestésicos associados, bloqueio neuromuscular com SQE $<0,9$ em $M_{1}$ e $M_{5}$ ou períodos de supressão no EEG, este avaliado pela taxa de supressão de surtos.

Conclui-se, então, que o BIS e SEF $_{95 \%}$, ambos obtidos de dois canais, podem ser utilizados na monitorização da profundidade da anestesia com sevoflurano em crianças ( 0 a 12 anos), observando-se os mesmos parâmetros sugeridos para adultos. Além disso, ficou evidente que a utilização de valores maiores que $1 \mathrm{CAM}$ são desnecessários para manter níveis adequados de hipnose, podendo esta ser conseguida com variações de 0,5 a 1 CAM, o que corresponde ao intervalo de 60 a 40 de BIS, respectivamente.

\section{Processed Electroencephalogram in Children Anesthetized with Sevoflurane. Is it Feasible?}

Rogean Rodrigues Nunes, TSA, M.D., Sara Lúcia Cavalcante, TSA, M.D., Alberto Esteves Gemal, TSA, M.D., Domingos Gerson de Sabóia Amorim, TSA, M.D.

\section{INTRODUCTION}

EEG-derived bispectral index (BIS) has been indicated as a major substrate for measuring hypnotic effects of anesthetic drugs in the central nervous system ${ }^{1.2}$. Many studies describe BIS as hypnotic depth indicator during inhalational anesthesia ${ }^{3}$, especially with sevoflurane ${ }^{4,5}$. BIS has been widely evaluated in adults ${ }^{4-10}$, but its pediatric usefulness is still controversial because current data are limited and questionable, especially in children aged 0 to 6 month ${ }^{11}$. Scher ${ }^{12}$ has shown that up to 6 months of age dominant EEG frequency is $5 \mathrm{~Hz}$; from 9 to 18 months it is $6-7 \mathrm{~Hz}$, at 2 years of age it is $7-8 \mathrm{~Hz}$; at 7 years of age it is $9 \mathrm{~Hz}$ and at 15 years of age it behaves as in adults: $10 \mathrm{~Hz}$.

No studies were found in the literature correlating a single anesthetic drug with EEG parameters in children.

This study aimed at evaluating changes in BIS, SEF $95 \%$ in channel 1 (left) and channel 2 (right), relative delta band frequency amplitude $(\delta \%)$ - (observing changes in cortical activity maturity) and suppression rate (SR) in children, adolescents and adults, both in deep hypnotic states and during emergence, and correlating these changes to sevoflurane pharmacodynamic variables: expired concentration (EC) and EC/MAC ratio for age.

\section{METHODS}

After the Hospital's Ethics Committee approval, participated in this study 100 patients (adults, adolescents and children) of both genders, aged 0 to 40 years, physical status ASA I and II, submitted to tonsylectomies, adenotonsylectomies, neurorrhaphies, tenorrhaphies, lower limb fractures correction and gynecological procedures. Exclusion criteria were heart, lung or neurological diseases, prematurity, obesity, or patients under drugs knowingly affecting EEG or sevoflurane's MAC. EEG data were measured with a specific device (Aspect A 1000 - release 3.31) ${ }^{\circledR}$, manufactured for adults. Two reference channels (F7 and F8) were used with reference $\mathrm{Fz}$ and ground $\mathrm{Fp} 2$, with silver/chloride leads in conducting gel coupled to the converter, being data computed after impedance test performed by the device itself and further approval, provided impedance recorded in all chan- 
nels was lower than $5 \mathrm{~K} \Omega$. Patients were distributed in 5 groups of 20, as follows: GI - 0 to 6 months; GII > 6 months to 2 years; GIII > 2 to 12 years; GIV > 12 to 18 years and GV > 18 to 40 years.

Patients were not premedicated and the presence of the mother in the OR was requested for groups I, II and III during anesthetic induction.

All patients were monitored in the OR with: sphygmomanometer for automatic systolic (SBP) and diastolic (DBP) blood pressure, heart rate $(\mathrm{HR})$, pulse oximetry for $\mathrm{SpO}_{2}$, capnography for $\mathrm{P}_{\mathrm{ET}} \mathrm{CO}_{2}$, anesthetic gases analyzer, cardioscopy in $\mathrm{D}_{\|}$and $\mathrm{V} 5$ leads, bispectral index (BIS), spectral edge frequency $95 \%\left(\mathrm{SEF}_{95 \%}\right)$ in two channels, relative delta band frequency amplitude in two channels ( $\delta \%)$, suppression rate (SR) and neuromuscular block monitoring by TOF stimulation at 14-second intervals using adductor pollicis (acceleromyography) after detection of supramaximal stimulation immediately before mivacurium injection. Intravenous puncture was performed after anesthetic induction in groups I, II and III. All patients were induced with sevoflurane and $60 \%$ nitrous oxide in oxygen through the tidal volume technique up to BIS 30 , when intravenous 80 $\mu \mathrm{g} . \mathrm{kg}^{-1}$ mivacurium was administered to help laryngeal mask insertion (after TOF 5\% T1). Next, mechanical ventilation was installed with volume loss compensating ventilator and nitrous oxide was withdrawn. Mechanical ventilation was adjusted for all patients to generate a tidal volume equivalent to $8 \mathrm{ml}^{\mathrm{kg}} \mathrm{kg}^{-1}$ and respiratory rate was adjusted to maintain $\mathrm{P}_{\mathrm{ET}} \mathrm{CO}_{2}$ between 35 and $45 \mathrm{mmHg}$. A circle system with $\mathrm{CO}_{2}$ absorber and $1 \mathrm{~L}$. $\mathrm{min}^{-1} \mathrm{O}_{2}$ flow was used and sevoflurane was vaporized through specific equipment. For clinical and statistical purposes, 5 moments were analyzed: $M_{1}$ - before anesthetic induction; $\mathrm{M}_{2}$ - BIS 60; $\mathrm{M}_{3}$ - BIS 50; $\mathrm{M}_{4}$ - BIS 40 and $\mathrm{M}_{5}$ $\mathrm{BIS}$ at emergence, considered when patients opened their eyes or attempted any coordinated movement.

General anesthesia to allow the above mentioned surgical procedures was induced immediately after $M_{5}$ in all groups, thus avoiding the influence of different surgical stimulations on hypnotic depth in all studied moments. Except for $\mathrm{M}_{1}, 5$ minutes was the standardized time for BIS adjustments and sevoflurane EC values were recorded 10 minutes after the stabilization of BIS values (40,50 and 60$)$. The following pa- rameters were also recorded for all moments: SBP, DBP, HR, BIS, SEF $95 \%$ in channels 1 and $2, \delta \%$, ST, EC, $\mathrm{SpO}_{2}$ and TOF. Variations of $\pm 25 \%$ in SBP, DBP and HR as compared to $M_{1}$ were not considered clinically significant.

Nasopharyngeal temperature was maintained between 36 and $37^{\circ} \mathrm{C}$ for all patients, with the help of forced convective thermal air blanket.

To check significant differences between mean variables, analysis of variance with repeated measures was used, followed by Tukey's test, when needed, being the latter applied to mean variables of groups within each moment and to mean variables of moments within each group, considering significant $p<0.05$.

\section{RESULTS}

Demographics data are shown in table I. Mean SBP, DBP and $\mathrm{HR}$, correlating moments within each group are shown in table II, figures 1, 2 and 3. Data show that SBP in all groups was significantly different in $M 3$ as compared to $M 1(p<0.05)$. However, except for groups IV and V, all other $M_{1}$ values were similar to $\mathrm{M}_{5}(\mathrm{p}>0.05)$ (Table III, Figures 5 and 6$)$. As to DBP, although not going beyond reference values for the age groups, moments $M_{2}, M_{3}$ and $M_{4}$ were significantly different from moments $M_{1}$ and $M_{5}$ in all groups, being $M_{5}$ significantly different from $M_{1}$ in group III only. As to HR, only group I had significant variations in all moments, being group II different only in moments $M_{1}, M_{4}$ and $M_{5}(p<0.05)$; and groups III, IV and V different in moments $M_{2}, M_{3}$ and $M_{4}(p<0.05)$, without, however, clinically significant differences. BIS analysis by group within each moment has shown that in moments $M_{1}, M_{2}, M_{3}$ and $M_{4}$, groups were not statistically different $(p>0.05)$. In moment $M_{5}$, groups I and IV had similar values but significantly different from groups II, III and V, without however extrapolating emergence-related values (Table III and Figure 4).

Power spectral analysis via SEF S5\% $_{9}$ in channels 1 and 2, related to moments within each group has shown that groups had statistically similar results in moments $M_{1}$ and $M_{5}$ only ( $p$ $>0.05$ ). (Table III and Figures 5 and 6).

Table I - Demographics Data

\begin{tabular}{|c|c|c|c|c|c|}
\hline \multirow[t]{2}{*}{ Variables } & \multicolumn{5}{|c|}{ Groups } \\
\hline & 1 & II & III & IV & V \\
\hline Age (years) * & $0.30 \pm 0.14$ & $1.26 \pm 0.51$ & $7.36 \pm 3.00$ & $15.3 \pm 1.74$ & $27.2 \pm 7.19$ \\
\hline Weight (kg) * & $5.37 \pm 1.06$ & $10.16 \pm 2.08$ & $24.32 \pm 7.43$ & $51.2 \pm 9.61$ & $65.75 \pm 4.89$ \\
\hline \multicolumn{6}{|c|}{ Physical status } \\
\hline ASA I & 17 & 15 & 15 & 10 & 14 \\
\hline ASA II & 03 & 05 & 05 & 10 & 06 \\
\hline \multicolumn{6}{|l|}{ Gender } \\
\hline Male & 13 & 12 & 18 & 11 & 12 \\
\hline Female & 07 & 08 & 02 & 09 & 08 \\
\hline
\end{tabular}

*Values expressed in Mean \pm SD 
Table II - Systolic Blood Pressure (SBP), Diastolic Blood Pressure (DBP) - (mmHg) and Heart Rate (HR)) - (beat.min $\left.{ }^{-1}\right)$, by Group within Each Moment, (Mean \pm SD)

\begin{tabular}{|c|c|c|c|c|c|c|}
\hline \multirow[t]{2}{*}{ Moments } & \multirow[t]{2}{*}{ Variables } & \multicolumn{5}{|c|}{ Groups } \\
\hline & & 1 & II & III & IV & $\mathrm{V}$ \\
\hline \multirow[t]{3}{*}{$\mathrm{M}_{1}$} & SBP & $80.30 \pm 1.30$ & $82.55 \pm 1.85$ & $92.20 \pm 11.33$ & $118.45 \pm 5.26$ & $120.10 \pm 6.99$ \\
\hline & DBP & $50.85 \pm 1.98$ & $61.95 \pm 1.36$ & $62.95 \pm 3.30$ & $74.45 \pm 4.39$ & $76.85 \pm 5.76$ \\
\hline & $\mathrm{HR}$ & $130.40 \pm 5.53$ & $121.70 \pm 4.50$ & $106.65 \pm 12.31$ & $82.60 \pm 5.29$ & $79.30 \pm 3.99$ \\
\hline \multirow[t]{3}{*}{$\mathrm{M}_{2}$} & SBP & $78.05 \pm 3.28$ & $81.15 \pm 2.66$ & $87.30 \pm 7.85$ & $109.90 \pm 3.88$ & $112.75 \pm 6.76$ \\
\hline & DBP & $45.70 \pm 2.25$ & $59.30 \pm 2.32$ & $61.65 \pm 2.23$ & $68.80 \pm 4.82$ & $67.70 \pm 3.20$ \\
\hline & $\mathrm{HR}$ & $124.90 \pm 4.93$ & $115.05 \pm 4.97$ & $99.85 \pm 10.04$ & $78.15 \pm 5.69$ & $73.65 \pm 3.47$ \\
\hline \multirow[t]{3}{*}{$M_{3}$} & SBP & $73.55 \pm 3.12$ & $77.85 \pm 2.11$ & $83.90 \pm 6.83$ & $100.30 \pm 2.85$ & $105.95 \pm 7.05$ \\
\hline & DBP & $46.40 \pm 2.46$ & $57.50 \pm 1.70$ & $58.20 \pm 3.74$ & $67.60 \pm 2.66$ & $64.00 \pm 2.71$ \\
\hline & $\mathrm{HR}$ & $120.20 \pm 7.42$ & $112.20 \pm 4.64$ & $95.70 \pm 13.08$ & $72.80 \pm 6.67$ & $67.50 \pm 5.37$ \\
\hline \multirow[t]{3}{*}{$\mathrm{M}_{4}$} & SBP & $70.95 \pm 3.07$ & $74.10 \pm 2.81$ & $79.30 \pm 5.87$ & $93.85 \pm 3.23$ & $98.45 \pm 1.99$ \\
\hline & SBP & $42.20 \pm 3.96$ & $52.00 \pm 4.09$ & $54.85 \pm 3.03$ & $64.30 \pm 3.87$ & $60.00 \pm 1.89$ \\
\hline & $\mathrm{HR}$ & $116.85 \pm 6.63$ & $105.30 \pm 4.53$ & $87.70 \pm 11.57$ & $66.40 \pm 6.64$ & $63.45 \pm 4.64$ \\
\hline \multirow[t]{3}{*}{$\mathrm{M}_{5}$} & SBP & $133.65 \pm 3.80$ & $64.50 \pm 1.50$ & $89.45 \pm 9.71$ & $114.95 \pm 2.82$ & $116.70 \pm 6.42$ \\
\hline & DBP & $133.65 \pm 3.80$ & $126.70 \pm 2.70$ & $69.05 \pm 8.64$ & $74.00 \pm 2.66$ & $79.65 \pm 3.77$ \\
\hline & $\mathrm{HR}$ & $133.65 \pm 3.80$ & $126.70 \pm 2.70$ & $109.60 \pm 12.71$ & $85.35 \pm 6.91$ & $79.90 \pm 5.48$ \\
\hline
\end{tabular}

Table III - Variables: BIS, SEF $95 \% 1(\mathrm{~Hz}), \mathrm{SE}_{95 \%} 2(\mathrm{~Hz}), \delta \%, \mathrm{EC}(\%)$ and EC/MAC by Group within Each Moment, (Mean \pm SD)

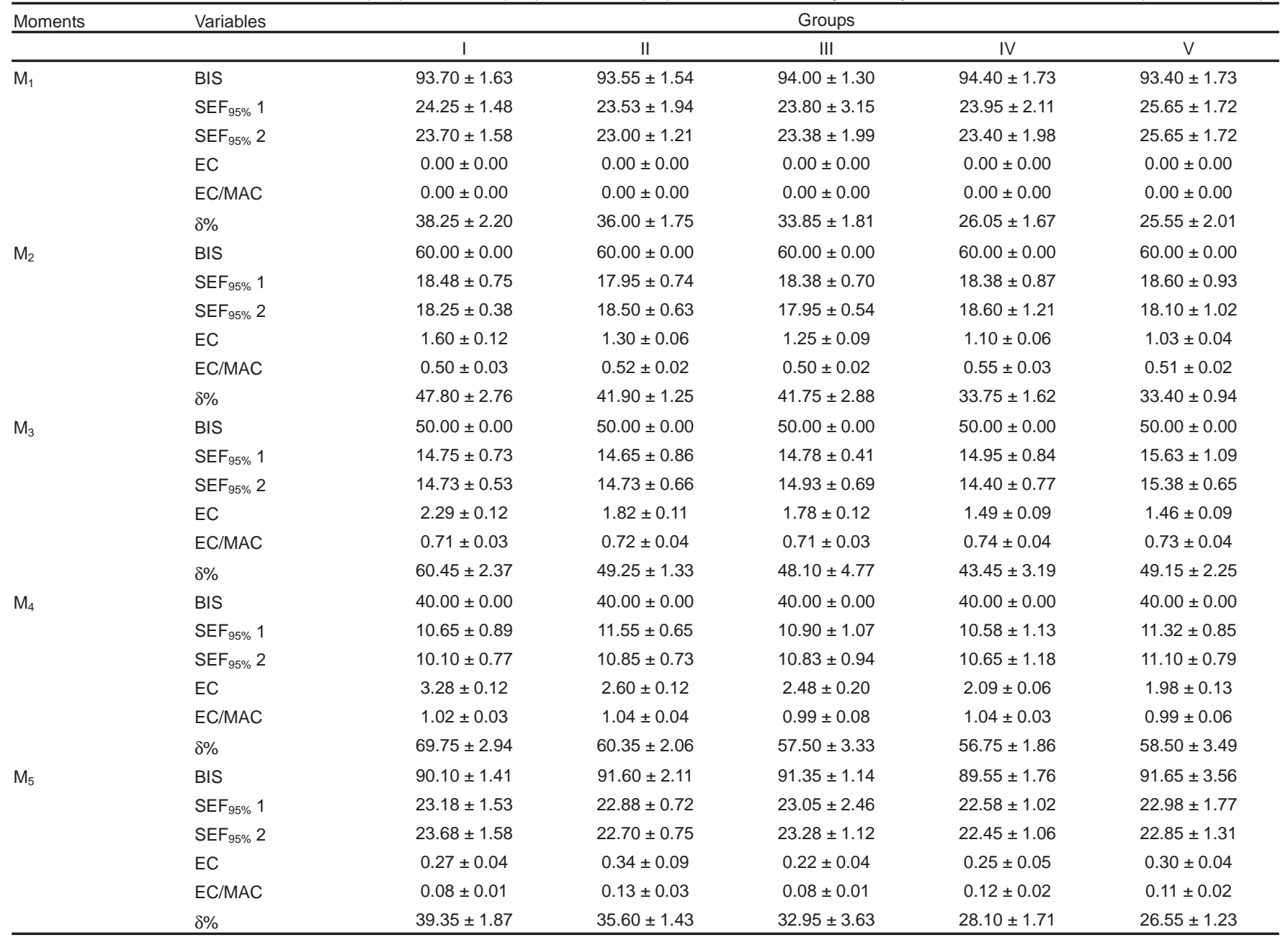




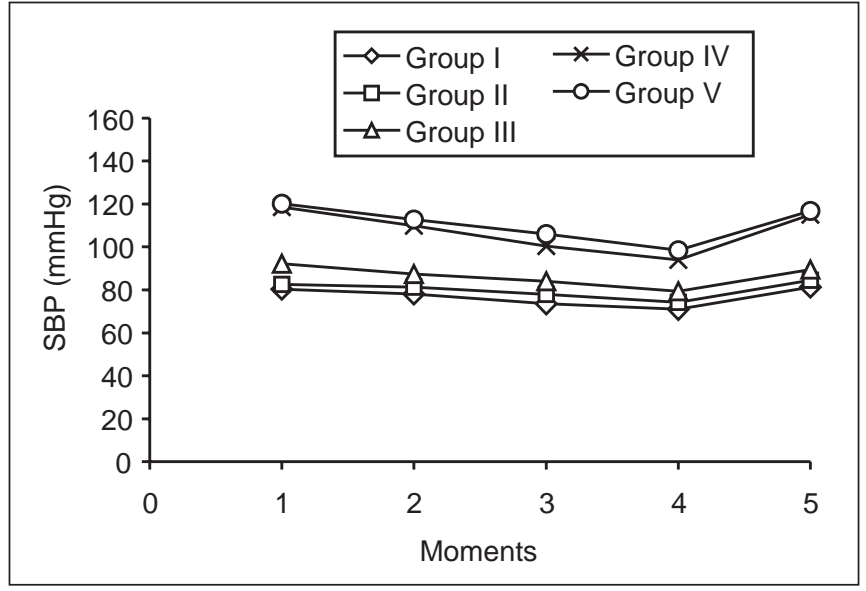

Figure 1 - Systolic Blood Pressure Variations (SBP) $(\mathrm{mmHg})$, by Moment within Each Group

$\left(p>0.05\right.$ for $M_{1}$ and $M_{5}$, except in groups IV and V) Tukey's test analysis

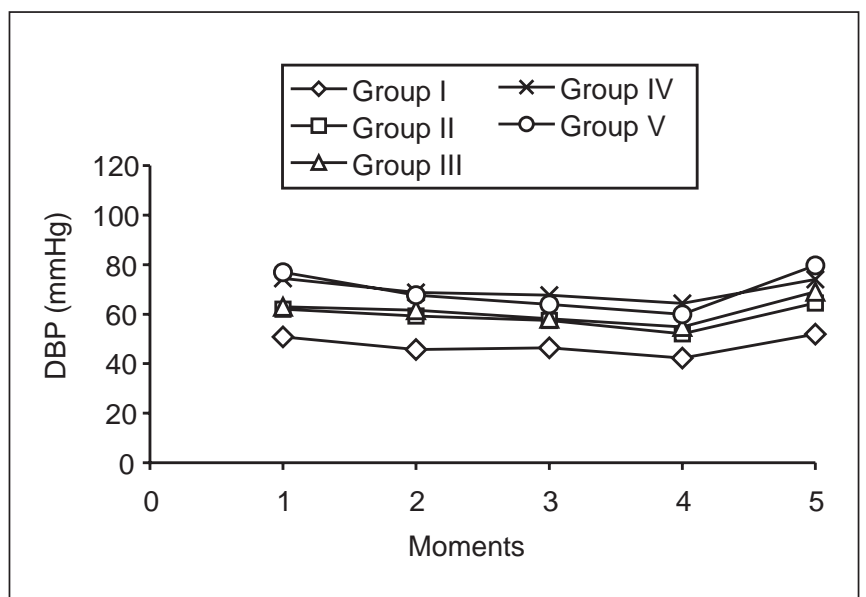

Figure 2 - Diastolic Blood Pressure Variations (DBP) $(\mathrm{mmHg})$, by Moment within Each Group

Moments $M_{2}, M_{3}$ and $M_{4}$ were significantly different from moments $M_{1}$ and $M_{4}$ in all groups - $(p<0.05)$. Tukey's test analysis

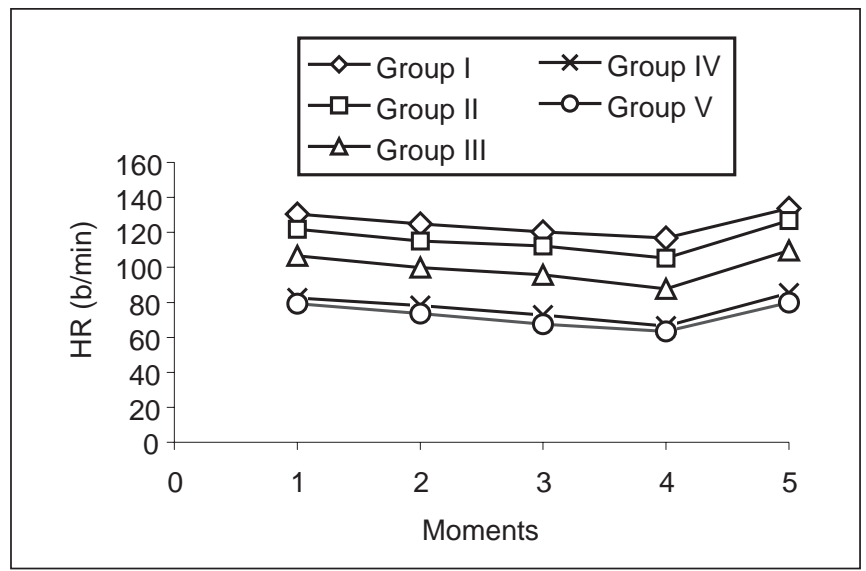

Figure 3 - Heart Rate Variations $(\mathrm{HR})(\mathrm{mmHg})$, by Moment within Each Group

$\left(p<0.05\right.$ in all group I moments; $p>0.05$ for moments $M_{1}$ and $\mathrm{M}_{5}$ in groups III, IV and V). Tukey's test analysis

Revista Brasileira de Anestesiologia

Vol. 53, № 3, Maio - Junho, 2003

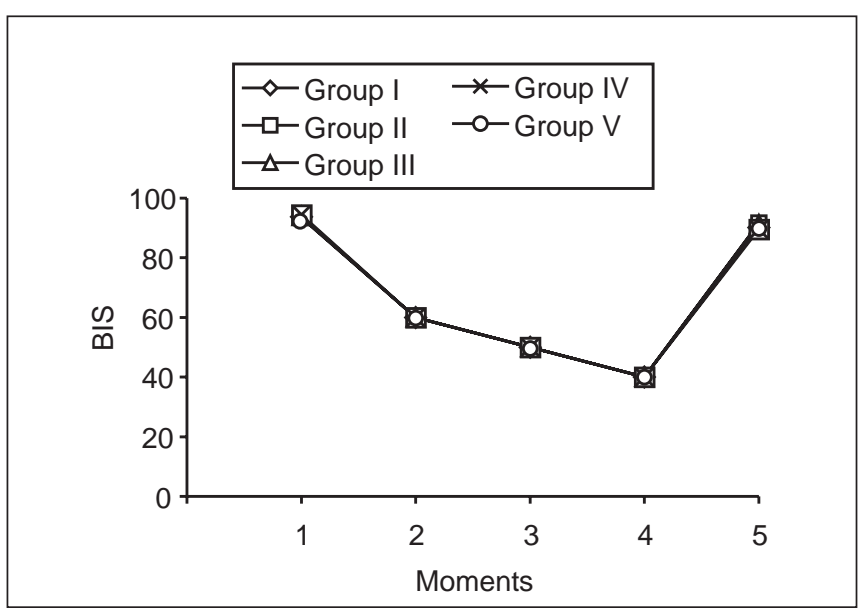

Figure 4 - Bispectral Index Variations (BIS)

$p>0.05$ in moments $M_{1}, M_{2}, M_{3}$ and $M_{4}$ analyzing all groups in each moment. Tukey's test analysis

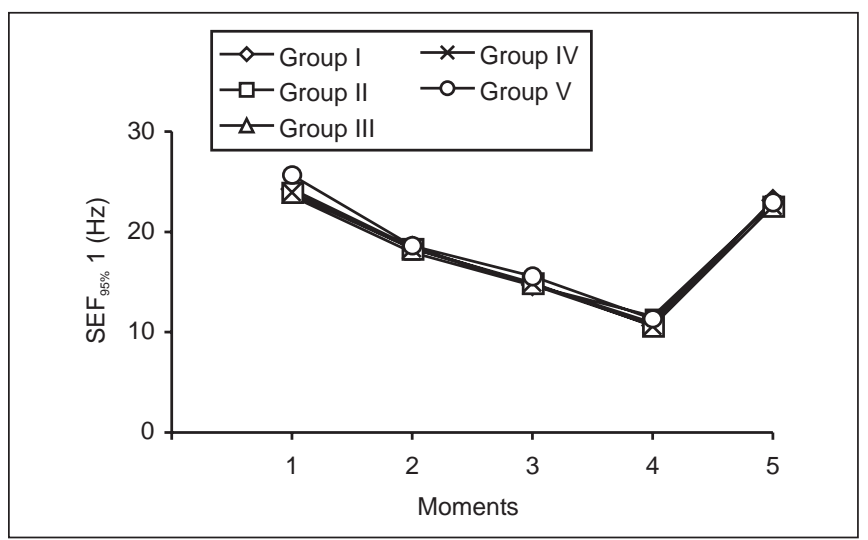

Figure $5-\mathrm{SEF}_{95 \%} 1$ Variations $(\mathrm{Hz})$

$p<0.5$ in moments $M_{1}$ and $M_{5}$, within each group. Tukey's test analysis

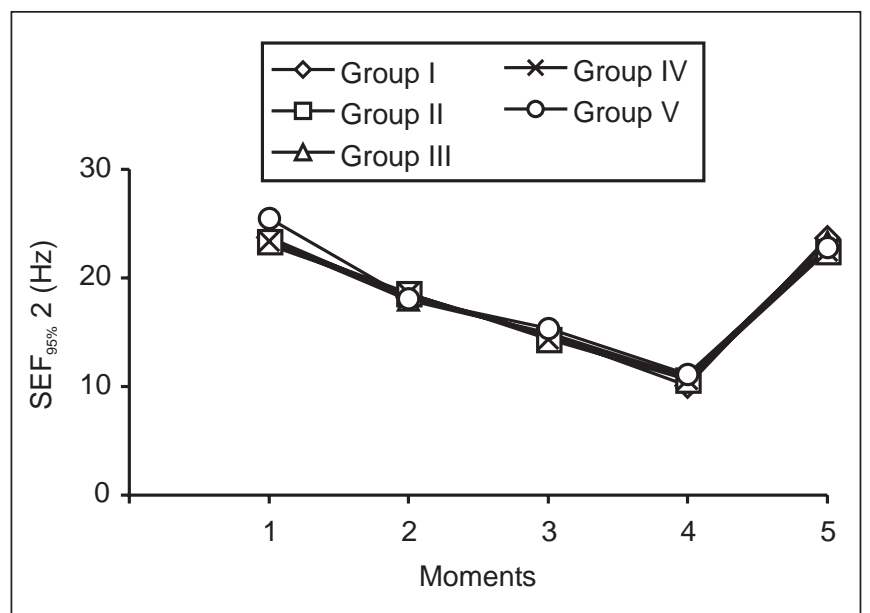

Figure $6-\mathrm{SEF}_{95 \%} 2$ Variation $(\mathrm{Hz})$

$p>0.05$ in moments $M_{1}$ and $M_{5}$ within each group. Tukey's test analysis 
Percentage analysis of delta frequency band resulting from channels 1 and 2 has shown that in all moments studied, group I values were statistically different from remaining groups, always with higher values $(p<0.05)$ (Table III and Figure 7). Expired concentrations observing groups within each moment have shown that in moments $M_{2}, M_{3}$ and $M_{4}$ groups II and III had similar results ( $p>0.05)$, however different from what has been observed in groups IV and V (similar among themselves). Nevertheless, values observed in moments $M_{2}$, $\mathrm{M}_{3}$ and $\mathrm{M}_{4}$ for group I were statistically different from all other groups, always with the highest scores $(p>0.05)$. For $\mathrm{M}_{5}$, all values were statistically different for all groups $(p<0.05)$. Mean values, however, have varied from $0.27 \%$ to $0.34 \%$. Pharmacokinetic data (EC and EC/MAC) and BIS are shown in table III and figures 8 and 9 . There were no statistically sig-

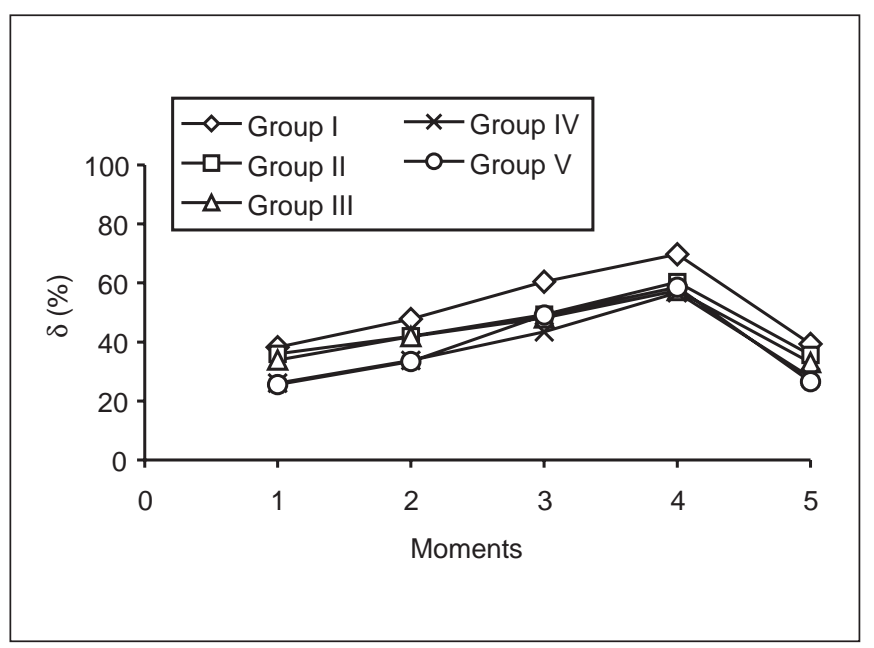

Figure $7-\delta \%$ Variations

$p<0.05$ in moments $M_{1}, M_{2}, M_{3}, M_{4}$ and $M_{5}$ within each group. Tukey's test analysis

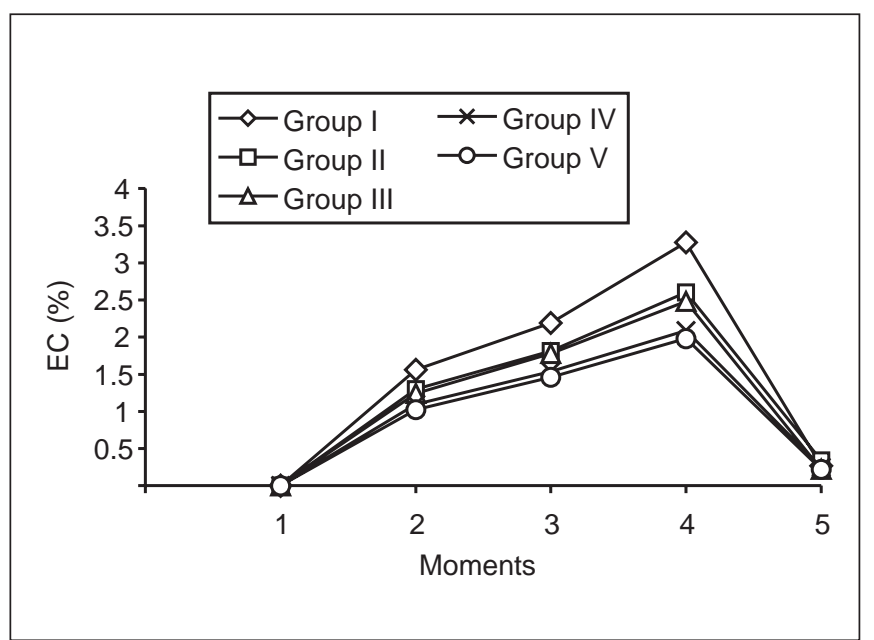

Figure 8 - Expired Concentration Variations (EC)

In analyzing groups within each moment we observed: $p>0.05$ for $M_{2}, M_{3}$ and $M_{4}$ in groups II and III. $p>0.05$ for $M_{2}, M_{3}$ and $M_{4}$ in groups IV and $\mathrm{V}$, with $\mathrm{p}<0.05$ when comparing groups II and III to groups IV and V in these same moments. $p<0.05$ for $\mathrm{M}_{2}$, $M_{3}$ and $M_{4}$ in group I as compared to other groups

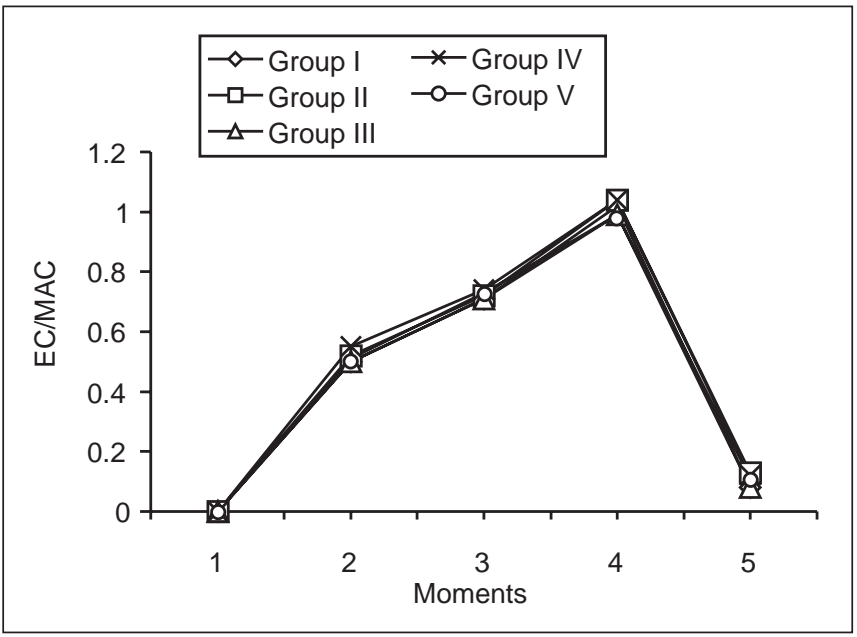

Figure 9 - EC/MAC Variations

Groups analysis within each moment. $p>0.05$ in $M_{2}, M_{3}$ and $M_{4}$

nificant differences in EC/MAC among groups in moments $M_{2}, M_{3}$ and $M_{4}(p>0.05)$. Emergence values $\left(M_{5}\right)$ however were statistically similar among groups II, IV and V, which were significantly different $(p<0.05)$ from groups I and III (similar among themselves). Whereas highestEC/MAC value is 0.13 (Group Il in $\mathrm{M}_{5}$ ), it only represents $43 \%$ of awaken MAC (0.3 MAC) for sevoflurane ${ }^{13}$. TS values were always zero in all moments studied. Before emergence, TOF would already indicate values above 0.9 for all patients.

\section{DISCUSSION}

It is unknown how processed EEG data (BIS and SEF $_{95 \%}$ ) could be different in children as compared to adults, especially children less than 6 months of age and possibly until 5 years of age, because brain maturation and synapses formation continue even after birth and go until 5 years of age, with most changes occurring in earlier moments of life ${ }^{14}$. However Denman et al., in a recent study evaluating children between 0 and 12 years of age ${ }^{15}$, have indicated that sevoflurane/BIS dose response ratio is similar in children breathing sevoflurane $/ \mathrm{N}_{2} \mathrm{O}$ as compared to adults breathing sevoflurane $/ \mathrm{O}_{2}{ }^{8}$, being sevoflurane concentration for BIS 50 $1.55 \%$ for children younger than 2 years of age and $1.25 \%$ for children between 2 and 12 years of age. In this study, however, Denman admits that preanesthetic medication has induced substantial sedation which was reflected in BIS values. In spite of this consideration, the study suggests that the same EEG equipment and method may be used in children. However Bannister et al. ${ }^{11}$ evaluating BIS monitoring effects in anesthesia and during anesthetic recovery in children, using sevoflurane and nitrous oxide, have shown that there are difficulties in adjusting sevoflurane concentrations to achieve a predetermined BIS value in patients between 0 and 6 months of age submitted to epidural caudal block. In addition, in this same study, BIS values remained low in spite of early sevoflurane withdrawal, what was not true for older paVol. 53, № 3, Maio - Junho, 2003 
tients. An explanation would be EEG differences in this population, determined by maturation and synapses formation during the first months of life ${ }^{16-18 .}$ Another explanation would be the presence of $60 \%$ nitrous oxide which, alone, could be enough to produce deep hypnosis in this population, concluding that further data are needed to interpret BIS in this age group. However, Bannister et al. have also introduced in the technique a potentially confusing factor in the comparison BIS/age: younger patients submitted to inguinal hernia correction were submitted to caudal block, which has not been the case with older patients submitted to tonsylectomies or adenoidectomies. This factor is potentially confusing because recent studies have shown a decrease in perioperative sedation in adults post regional blockade ${ }^{19,20}$. In addition, authors have not referred BIS values at emergence in this population. Davidson et al. ${ }^{21}$ in a recent study evaluating BIS differences in children below 15 years of age and induced with sevoflurane and nitrous oxide in oxygen under mask, during emergence after postectomies (53 patients), have shown that BIS immediately before emergence tends to be lower in children between 5 and 11 months of age $(67.8 \pm 10)$ as compared to children between 12 and 178 months of age (73.5 \pm 7 ), and that after emergence, mean BIS values were: children between 5 and 11 months of age $(85.6 \pm 13.6)$ and children between 12 and 178 months of age $(83.1 \pm 12)$, concluding that BIS may be applied to children as it is applied to adults, but its validation in the group between 5 and 11 months deserves further studies. The authors, however, have investigated BIS values under low sevoflurane concentrations, that is, superficial anesthesia, without showing a clear relationship between BIS and anesthetic expired concentration, which was fixed in $0.9 \%, 0.7 \%$ and $0.5 \%$, without considering MAC variations in different age groups ${ }^{22}$, which may result in major variations in BIS and SEF $_{95 \%}$ values. Glass et al. ${ }^{4}$ have shown loss of consciousness with a mean BIS of 71 in patients anesthetized with isoflurane. Katot et al. ${ }^{8}$ have shown that BIS values in adults sedated with sevoflurane who responded to oral commands was 73. Davidson et al. have shown that BIS in younger children ( 5 to 11 months) should be carefully interpreted because although there is a significant BIS increase in this group after stimulated emergence, they have a lower BIS as compared to older children (12 to 178 months) immediately before emergence, in addition to having a wide BIS variation. A correlation has also not been shown between sevoflurane expired concentration and BIS during emergence, which contrasts with a recent study by Denman et al. ${ }^{15}$. Moreover, this study by Davidson et al. has maintained ventilation under mask during surgery, what impairs respiratory rate and tidal volume regularity, as well as a more adequate balance in sevoflurane expired concentration values.

Degout et al. ${ }^{23}$ in a recent study evaluating BIS and hypnotic components of sevoflurane-induced anesthesia, and comparing children aged 3.5 to 13 years to adults aged 22 to 72 years, have shown that BIS has a good correlation with hypnotic components of anesthesia in children and adults induced with sevoflurane. In this study, however, authors have premedicated all patients with sedatives, in addition to bolus alfentanil before tracheal intubation, and continuous infusion throughout the surgery, what directly changes the evaluation of sevoflurane/BIS correlation.

Johansen et al. ${ }^{24}$ in a continuous evaluation of preoperative BIS and comparing postoperative results in children aged 2 to 12 years have shown that BIS 85 represents an adequate emergence for extubation and that BIS may be a useful tool for pediatric anesthesia.

Our study has evaluated the correlation of pharmacodynamic and EEG parameters using sevoflurane, which is an inhalational agent with low blood/gas partition coefficient, not pungent, not flammable and with limited cardiopulmonary depression, which may be useful for adults and children ${ }^{25-27}$. Previous studies with halothane, isoflurane and desflurane have shown that MAC increases with decreased age. Sevoflurane's pharmacology in neonates and children has been accurately determined in a study by Lerman et al. ${ }^{22}$ who showed that mean MAC in neonates is $3.2 \%$; in children $>1$ up to 6 months is $3.2 \%$; in children $>6$ up to 12 months is $3.2 \%$, and in children $>1$ up to 12 years is $2.5 \%$. Sevoflurane MAC in adults is $2 \%{ }^{13}$.

The correlation between different groups/moments in our study was: Group I (0 to 6 months): $\mathrm{M}_{1}$ (awaken) has shown that mean BIS values are compatible with adult awaken and conscious patients. In addition, SEF $_{95 \%}$ values in both channels were also correlated to awaken patients. However, in the percentage analysis of spectral density $(0-30 \mathrm{~Hz})$ in delta frequency band, it was higher as compared to adults, in line with Scher ${ }^{12}$ findings, where the author states that in this age group the predominant frequency in awaken patients is between 1-5 Hz, which has not influenced spectral edge or BIS values, as compared to adults. In $\mathrm{M}_{2}$ (BIS 60), patients no longer responded to tactile or verbal commands. SEF $95 \%$ was decreased in both channels and there was an increase in spectral density percentage in delta frequency band, what is compatible with the deepening of anesthesia. These changes appeared with a mean sevoflurane EC value of $1.6 \%$ and mean $\mathrm{EC} / \mathrm{MAC}$ equal to 0.5 . In $\mathrm{M}_{3}$ (BIS 50) there has been a further decrease in $\mathrm{SEF}_{95 \%}$ (both channels) as well as in $\delta \%$ with mean EC of $2.29 \%$ and mean EC/MAC of 0.71 , showing further deepening of anesthesia. Lower SEF $_{95 \%}$ values were observed in $\mathrm{M}_{4}$ (BIS 40), as well as higher $\delta \%$ va- lues, which is still compatible with further deepening of anesthesia (electric activity slowdown, with increased spectral power in low frequency bands). Mean EC value was $3.28 \%$ and mean EC/MAC value was 1.02. A regression of EEG parameters (SEF ${ }_{95 \%}$, BIS and $\delta \%$ ) was seen in $\mathrm{M}_{5}$ (emergence), which have returned to values close to baseline. We have not evaluated emergence time because we believe that the most important thing would be the analysis of the correlation between EEG values and clinically pre-established emergence data. Emergence time evaluation was confusing in Bannister et al. ${ }^{11}$ study with patients aged 0 to 6 months submitted to inguinal hernia correction using general/regional anesthesia and nitrous oxide, showing that although major decreases in sevoflurane administration during surgery, BIS values re- 
mained below 40. This however was not clear in the clini$\mathrm{cal} / \mathrm{EEG}$ correlation evaluation, in addition to suggesting that $60 \%$ nitrous oxide alone would be enough to maintain deep hypnosis in this pediatric population.

Group II (6 months to 2 years): in $\mathrm{M}_{1}$ (awaken), BIS and $\mathrm{SEF}_{95 \%}$ values in channels 1 and 2 were also similar to adults (awaken). As to moments $\mathrm{M}_{2}, \mathrm{M}_{3}, \mathrm{M}_{4}$ and $\mathrm{M}_{5}$, BIS and SEF $\mathrm{S}_{95}$ variations behaved similarly to groups I, III, IV and V. Absolute EC values, however, moment by moment, increased as BIS and $S F_{95 \%}$ values decreased, but EC values relative to MAC (EC/MAC) for this age group remained similar to group I, moment by moment. As to $\delta \%$, there has been spectral density decrease, which may be related to brain maturation evolution. EEG data (BIS, $\mathrm{SEF}_{95 \%}$ and $\delta \%$ ) returned to baseline values $\left(\mathrm{M}_{1}\right)$ in moment $\mathrm{M}_{5}$.

Group III (2 to 12 years): EEG variables analysis (BIS, $\mathrm{SEF}_{95 \%}$ and $\delta \%$ ) as well as EC and EC/MAC were similar to group II in all moments. There was, however, a mean spectral density decrease in delta frequency band in all moments as compared to group II.

Groups IV (12 to 18 years) and V (18 to 40 years) behaved similarly in all moments for BIS, SEF $95 \%$, EC and EC/MAC, being also shown that for BIS 40, sevoflurane EC for both groups was similar to $1 \mathrm{MAC}$, relationship maintained in all studied groups in BIS 40. In addition, it has been observed that $\delta \%$ in both groups behaved similarly in all moments. In $\mathrm{M}_{1}$, spectral densities were lower in percentage as compared to previous groups, configuring a shift of spectral power toward higher frequencies in this moment. Global analysis in this study has shown that there were major changes in spectral densities with regard to $\delta \%$, which decreased as age increased in awaken patients in all groups. However, spectral variations during anesthesia have behaved similarly for all groups, that is, there has been an increase in delta frequency band spectral density $(0-5 \mathrm{~Hz})$ in anesthetized patients, with higher absolute values recorded for group I. SEF $95 \%$ in channels 1 and 2 were similar among adults adolescents and children in all moment. BIS values, predetermined or not, related to sevoflurane pharmacodynamic data, have shown a direct relationship with MAC multiples (EC/MAC) in different age groups in all moments and these data were validated without the influence of: preanesthetic medication, nitrous oxide, surgical stimulations, associated anesthetic blocks, neuromuscular block with TOF $<0.9$ in $\mathrm{M}_{1}$ and $\mathrm{M}_{5}$ or EEG suppression periods evaluated by suppression rate.

The conclusion is that BIS and SEF $_{95 \%}$, both obtained from two channels, may be used to monitor sevoflurane anesthetic depth in children (0 to 12 years), observing the same parameters suggested for adults. In addition, it was clear that values above 1 MAC are unnecessary to maintain adequate hypnotic levels, which may be obtained with variations of 0.5 to 1 MAC, corresponding to the interval of BIS 60 to 40, respectively.

\section{REFERÊNCIAS - REFERENCES}

01. Sigl JC, Chamoun NC - An introduction to bispectral index analysis for the EEG. J Clin Monit, 1994;10:392-404.

02. Liu J, Singh H, White P - Electroencephalographic bispectral index correlates with intraoperative recall and depth of propofol-induced sedation. Anesth Analg, 1997;84:185-189.

03. Nunes RR, Cavalcante SL, Zeferino T - Influência da clonidina na anesthesia inalatória com sevoflurano em adultos. Avaliação pelo índice bispectral. Rev Bras Anestesiol, 1999;49:89-93.

04. Glass PS, Bloom M, Kearse L et al - Bispectral analysis measures sedation and memory effects of propofol, midazolam, isoflurane and alfentanil in healthy volunteers. Anesthesiology, 1997;86:836-847.

05. Kearse LA, Rosow C, Zaslavsky A et al - Bispectral analysis of the electroencephalogram predicts conscious processing of information during propofol sedation and hypnosis. Anesthesiology, 1998:88:25-34.

06. Flaishon R, Windsor A, Sigl J et al - Recovery of consciousness after thiopental or propofol. Anesthesiology, 1997;86:613-619.

07. Gan TS, Glass PS, Windsor A et al - Bispectral index monitoring allows faster emergence and improved recovery from propofol, alfentanil and nitrous oxide anesthesia. Anesthesiology, 1998;88:642-650.

08. Katoh T, Suzuki A, Ikeda K - Electroencephalographic derivatives as a tool for predicting the depth of sedation and anesthesia induced by sevoflurane. Anesthesiology, 1998;88:642-650.

09. Song D, Joshi G, White PF - Tritation of volatile anesthetics using bispectral index facilitates recovery after ambulatory anesthesia. Anesthesiology, 1997;87:842-848.

10. Liu J, Singh H, White PF - Electroencephalographic bispectral analysis predicts the depth of midazolam induced sedation. Anesthesiology, 1996;84:64-69.

11. Bannister CF, Brosius KK, Sigl JC et al - The effect of bispectral index monitoring on anesthetic use and recovery in children anesthetized with sevoflurane in nitrous oxide. Anesth Analg, 2001;92:877-881.

12. Scher MS - Pediatric Neurophysiologic Evaluation, em: Swaiman KF, Ashwal S - Pediatric Neurology. St. Louis, Mosby, 1999;142-181.

13. Marshall BE, Longnecker DE - General Anesthetics, em: Hardman JG, Limbird LE, Molinoff PB et al - Goodman \& Gilman's. The Pharmacological Basis of Therapeutics. $9^{\text {th }} \mathrm{Ed}$, New York, McGraw-Hill, 1996;307-330.

14. Witte $\mathrm{H}$, Putsche $\mathrm{P}$, Eiselt $\mathrm{M}$ et al - Multimodal time-variant signal analysis of neonatal EEG burst patterns. Medinfo, 1998;9: 1250-1254.

15. Denman W, Swanson EL, Rosow D et al - Initial pediatric evaluation of the bispectral index (BIS) monitor and correlation of BIS with end-tidal sevoflurane concentration in infants and children. Anesth Analg, 2000;90:872-877.

16. Torres F, Anderson C - The normal EEG of the human newborn. J Clin Neurophysiol, 1995;2:89-103.

17. Holmes GL, Lombrosco CT - Prognostic value of background patterns in the neonatal EEG. J Clin Neurophysiol, 1993;10: 323-352

18. Scher MS, Sun M, Hatzilabrou GM et al - Computer analysis of EEG-sleep in the neonatal. J Clin Neurophysiol, 1990;7: 417-441.

19. Tverskoy M, Fleyshman G, Bachrak L et al - Effect of bupivacaine-induced spinal block on the hypnotic requirement of propofol. Anaesthesia, 1996;51:652-653. 
20. Tverskoy M, Shifrin V, Finger $J$ et al - Effect of epidural bupivacaine block on midazolam hypnotic requirements. Reg Anesth Pain Med, 1996;21:209-213.

21. Davidson AJ, McCann M, Devavaram P et al - The differences in the bispectral index between infants and children during emergence from anesthesia after circumcision surgery. Anesth Analg, 2001;93:326-330.

22. Lerman J, Sikich N, Kleinman S et al - The pharmacology of sevoflurane in infants and children. Anesthesiology, 1994;80: 814-824

23. Degout CS, Macabeo C, Dubreuil C et al - EEG bispectral index and hypnotic component of anaesthesia induced by sevoflurane: comparison between children and adults. $\mathrm{Br} \mathrm{J}$ Anaesth, 2001;86:209-212.

24. Johansen JW - Continuous intraoperative bispectral index monitoring and perioperative outcome in children. Anesth Analg, 1998;86:406S.

25. Naito $Y$, Tamai S, Shingu $\mathrm{K}$ et al - Comparison between sevoflurane and halothane for paediatric ambulatory anaesthesia. Br J Anaesth, 1991;67:387-389.

26. Strum DP, Eger II EI - Partition coefficients for sevoflurane in human blood, saline, and olive oil. Anesth Analg, 1987;66: 654-656.

27. Yasuda N, Targ AG, Eger II EI - Solubility of I-653, sevoflurane, isoflurane and halothane in human tissues. Anesth Analg, 1989;69:370-373.

\section{RESUMEN}

Nunes RR, Cavalcante SL, Gemal AE, Amorim DGS - Electroencefalograma Procesado en Niños Anestesiados con Sevoflurano. ¿Es Posible?

JUSTIFICATIVA Y OBJETIVOS: El índice bispectral (BIS), ha sido indicado como un substrato importante en la medición del efecto hipnótico de drogas anestésicas. No obstante, existen apenas dados limitados del uso del EEG en niños durante anestesia. El objetivo de este estudio es evaluar, en niños, los cambios en el BIS, SEF $F_{95 \%}$, amplitud relativa en la banda de frecuencia delta ( $\delta \%)$ y tasa de supresión de ataques (TS), correlacionando con variables farmacodinámicas del sevoflurano (CE y CE/CAM), comparándolas con el adulto.

MÉTODO: Participaron del estudio, 100 pacientes de ambos sexos, con edades entre 0 y 40 años, estado físico ASA I y II. Todos los pacientes fueron inducidos con sevoflurano siendo utilizado bloqueador neuromuscular cuando el BIS llegó a 30, siendo estratificados en 5 grupos: GI (20) - edad entre 0 y 6 meses; GII (20) - edad > 6 meses hasta 2 años; GIII (20) - edad $>2$ años hasta 12 años; GIV (20) - edad > 12 años hasta 18 años y GV (20) - edad > 18 años hasta 40 años. En cada grupo 5 momentos fueron evaluados: $M_{1}$ (alerta); $M_{2}$ (BIS 60); $M_{3}$ (BIS 50): $M_{4}$ (BIS 40) y $M_{5}$ (despertar), siendo, en todos los momentos, anotados los siguientes parámetros: PAS, PAD, $F C, B I S, S E F_{95 \%}, \delta \%$, tasa de supresión de ataques, $C E$ y CE/CAM.

RESULTADOS: LoS valores de BIS y SEF $F_{95 \%}$ presentaron correlación directa con la CE/CAM del sevoflurano a valores de BIS de 40,50, 60 y despertar, respetándose la CAM para edad $(p>0,05)$. A $\delta \%$, en el $G /$ presentó valores superiores a todos los otros grupos, en los cinco momentos $(p<0,05)$.

CONCLUSIONES: EI BIS y SEF S5\% $_{9}$ pueden ser utilizados en la monitorización de la profundidad de la anestesia con sevoflurano en niños de 0 a 12 años observándose los mismos parámetros sugeridos para adultos. Lo mismo no sucede con la $\delta \%$, el cual mostró variaciones dependientes, probablemente, de la maduración cerebral. 